\title{
Comparative Evaluation of Four Bacteria-Specific Primer Pairs for $16 S$ rRNA Gene Surveys
}

\author{
Sofie Thijs ${ }^{1 * t}$, Michiel Op De Beeck ${ }^{2}$, Bram Beckers ${ }^{1}$, Sascha Truyens ${ }^{1}$, Vincent Stevens ${ }^{1}$, \\ Jonathan D. Van Hamme ${ }^{3}$, Nele Weyens ${ }^{1}$ and Jaco Vangronsveld ${ }^{1}$ \\ ${ }^{1}$ Centre for Environmental Sciences, Hasselt University, Diepenbeek, Belgium, ${ }^{2}$ Department of Biology, Lund University, \\ Lund, Sweden, ${ }^{3}$ Department of Biological Sciences, Thompson Rivers University, Kamloops, BC, Canada
}

OPEN ACCESS

Edited by:

Etienne Yergeau,

University of Quebec, Canada

Reviewed by:

Alessio Mengoni

University of Florence, Italy

Julien Tremblay

National Research Council Canada

(CNRC), Canada

*Correspondence:

Sofie Thijs

sofie.thijs@uhasselt.be

${ }^{\dagger}$ Present Address:

Sofie Thijs,

Hasselt University, Centre for Environmental Sciences, Diepenbeek, Belgium

Specialty section:

This article was submitted to

Terrestrial Microbiology,

a section of the journal

Frontiers in Microbiology

Received: 27 December 2016 Accepted: 09 March 2017

Published: 28 March 2017

Citation:

Thijs S, Op De Beeck M, Beckers B, Truyens S, Stevens V, Van Hamme JD, Weyens N and Vangronsveld J (2017)

Comparative Evaluation of Four Bacteria-Specific Primer Pairs for 165 rRNA Gene Surveys.

Front. Microbiol. 8:494. doi: 10.3389/fmicb.2017.00494
Bacterial taxonomic community analyses using PCR-amplification of the 16S rRNA gene and high-throughput sequencing has become a cornerstone in microbiology research. To reliably detect the members, or operational taxonomic units (OTUs), that make up bacterial communities, taxonomic surveys rely on the use of the most informative PCR primers to amplify the broad range of phylotypes present in up-to-date reference databases. However, primers specific for the domain Bacteria were often developed some time ago against database versions that are now out of date. Here we evaluated the performance of four bacterial primers for characterizing complex microbial communities in explosives contaminated and non-contaminated forest soil and by in silico evaluation against the current SILVA123 database. Primer pair 341f/785r produced the highest number of bacterial OTUs, phylogenetic richness, Shannon diversity, low non-specificity and most reproducible results, followed by 967f/1391r and 799f/1193r. Primer pair 68f/518r showed overall low coverage and a bias toward Alphaproteobacteria. In silico, primer pair 341f/785r showed the highest coverage of the domain Bacteria (96.1\%) with no obvious bias toward the majority of bacterial species. This suggests the high utility of primer pair 341f/785r for soil and plant-associated bacterial microbiome studies.

Keywords: pyrosequencing, 16S rRNA gene sequence primers, microbial communities, soil, explosives contamination

\section{INTRODUCTION}

High-throughput amplicon sequencing of taxonomic marker genes has become a widely used technique to obtain information on the microbial community composition, diversity, and richness at high resolution in diverse environmental samples (Caporaso et al., 2011; Yergeau et al., 2012a,b). This information has led to significantly increased understanding of the effect of natural or humaninduced disturbance on soil and plant-associated microbial communities (Hart et al., 2015; Yergeau et al., 2015; Beckers et al., 2016). While there is no doubt that short-read sequencing technologies have opened up a new dimension of microbial biodiversity research, the technologies also add complexity to the experimental study design. One of the most critical steps for accurate prokaryotic rRNA gene amplicon analyses is the choice of primers.

Primers designed for $16 \mathrm{~S}$ rDNA usually target a single stretch of the hypervariable regions of the 16S rRNA gene, allowing for species-level taxonomic classification in some cases 
(Baker et al., 2003; Lundberg et al., 2013). Selection of the Vregion targeted and associated primer pairs should therefore be done with care to ensure coverage of relevant microbial taxa and accurate representation of the microbiota profiles in microbiome analyses (Berry et al., 2011; Soergel et al., 2012; Ghyselinck et al., 2013; Walters et al., 2016). Using suboptimal primer pairs can lead to under-representation of certain taxa or selection against single species which can lead to questionable biological conclusions. Moreover, amplification of non-target sequences can consume unnecessary sequencing resources.

Recent attempts have been concerned with optimizing the prokaryotic primer set 515f/806r to target both Bacteria and Archaea (Apprill et al., 2015; Parada et al., 2015; Walters et al., 2016). While this primer pair, used traditionally by the Earth Microbiome Project (Gilbert et al., 2014), is indisputably important in soil ecology studies, it is also crucially important to continue to test and validate other primer sets ensuring that we can continue to uncover the wealth of information that is present in microbial communities. In some studies, it is even more desirable to focus on bacteria alone to centralize the effort and expenses to optimally address the research question. For example, in the field of bioremediation, several reports focus on re-structuring bacterial communities to improve the efficiency of organic contaminant degradation (Bell et al., 2013a,b; Thijs et al., 2016), monitor bacterial community structure in response to pollution (Truyens et al., 2013; Bell et al., 2015), or in response to agricultural management (Jenkins et al., 2016), plant genetic modification (Beckers et al., 2016), and to study the connections between plant-associated bacteria and human/animal microflora (Berg et al., 2015; Mahnert et al., 2015). For all these studies, next to universal prokaryotic primers, adequate bacteria-specific primers that are carefully selected in terms of coverage, reproducibility, exclusion of the amplification of host-organelles, and comparability between studies is needed.

Plant-associated microbiome studies often rely on the use of primers tested against databases from some years ago. In an extensive study by Klindworth et al. (2013), 175 broadrange $16 \mathrm{~S}$ rRNA gene primers and 512 primer pairs were tested in silico against the 376437 16S/18S rRNA gene sequences in the SILVA v108 database for amplification of bacteria and archaea sequences. However, since 2013, an additional 222,034 sequences have been added to the SILVA database, including new microbial lineages with potentially poor or sub-optimal binding of existing PCR-primers (Quast et al., 2013; Hug et al., 2016). It is therefore important that in light of the ongoing refinements to microbial phylogeny and taxonomy and the increased use of high-throughput sequencing technologies, up-to-date and broadly applicable bacterial primers are re-evaluated to assure the readiness for rational exploration of bacterial diversity and community structure.

Bacteria play an important role in organic compound transformation including the breakdown and detoxification of organic contaminants. Soil bacterial communities have therefore been the subject of bioremediation studies aiming to optimize bacterial community function for accelerating biodegradation and soil remediation (Yang et al., 2012; Yergeau et al., 2012a, 2015). Primer pair 341f/785r used by Klindworth et al. (2013) has been used on a variety of sample types, from marine samples to human samples (Hiergeist et al., 2016), but until now it has not been thoroughly tested on soil. Other primer pairs targeting the V3-V4 region, namely 341f/533r (Yergeau et al., 2014) and 343f/806r (Yergeau et al., 2015) have shown to amplify diverse bacterial communities in the rhizosphere of Salix purpurea, suggesting the utility of V3-V4 primer pairs for plant microbiome research.

When amplifying regions of the 16S rRNA gene from soil and plant-associated samples, it is also important to reduce the amplification of non-target DNA-sequences such as those coextracted from chloroplasts and mitochondria. To account for this, a chloroplast mismatch primer such as $799 \mathrm{f}$ (Chelius and Triplett, 2001) may be included. Alternatively, the use of peptidenucleic acid (PNA) PCR-clamps has been suggested (Lundberg et al., 2013). However, the effectiveness of both approaches to reduce host-organelle amplification depends largely on the plant species. In addition, modifications in the primer pair to reduce chloroplast amplification can also introduce new biases against bacterial groups which are abundant in soil (Lundberg et al., 2012). It is therefore important to carefully compare the performance of primer pairs in terms of taxonomic coverage and non-target amplification to gain the most out of the sequencing effort.

The objective of this study was to test the experimental applicability of four bacteria-specific primer pairs in order to maximize amplification efficiency, taxonomic coverage and target specificity. In contrast to other studies focusing on the evaluation of universal 16S rRNA gene primers, we focus here on the evaluation and observation of the bacterial community in more detail. Inspired by the study of Klindworth et al. (2013), we included primer pair $341 \mathrm{f} / 785 \mathrm{r}$ in addition to three other common primer sets spanning different regions of the $16 \mathrm{~S}$ rRNA gene. The primer pairs were tested empirically on a 2,4,6trinitrotoluene (TNT)-contaminated soil, a non-contaminated forest bulk soil and Acer pseudoplatanus rhizosphere soil, habitats known to harbor diverse sets of bacteria. Additionally, the primer pairs were evaluated in silico against the SILVA v123 database, the latest version available at time of writing. The results showed significant differences in taxonomic coverage, diversity, reproducibility, exclusion of chloroplast and ability to discriminate between polluted and non-polluted soils, depending on the primer pair used. We found that $341 \mathrm{f} / 785 \mathrm{r}$ detected the highest bacterial diversity, broadest taxonomic coverage, and provided the most reproducible results. Additionally, TNT was found to significantly alter soil bacterial community structure and change the relative abundance of several OTUs.

\section{MATERIALS AND METHODS}

\section{Study Site and Soil Sampling}

Soil samples were collected from a military TNT-production facility in Zwijndrecht, Belgium $\left(51^{\circ} 11^{\prime} 40.0^{\prime \prime} \mathrm{N} ; 4^{\circ} 19^{\prime} 29.6^{\prime \prime} \mathrm{E}\right)$ on July 8th 2013. The soil was a loamy sand with a moderately thick litter layer and humus with undecomposed and partly degraded organic matter. The TNT-contaminated soil had an average $\mathrm{pH}$ of 6.1 , moisture content of $66.2 \%$, conductivity of 340 $\mu S$ per $\mathrm{cm}$, cation exchange capacity of $9.2 \mathrm{Meq}$ per $100 \mathrm{~g}$ dry weight using the ammonium acetate method at $\mathrm{pH} 7$ (Chapman, 
1965) and $3.1 \mathrm{mg}$ organic labile carbon per kg dry weight soil measured using the permanganate oxidation method (Culman et al., 2012). The non-contaminated soil, a few meters away from the TNT-contaminated location, had an average $\mathrm{pH}$ of 7.5 , moisture content of $24 \%$, conductivity of $418 \mu \mathrm{S}$ per $\mathrm{cm}$, cation exchange capacity of $9.5 \mathrm{Meq}$ per $100 \mathrm{~g}$ dry weight and $1.6 \mathrm{mg}$ organic labile carbon per kg dry weight soil. Extraction of explosives from soil was performed according to EPA-method 8330. HPLC analyses were performed on an Agilent 1100 system (Agilent, Santa Clara, CA) with Chromosphere C18 reverse phase column $(5 \mu \mathrm{m}, 250 \times 4.6 \mathrm{~mm})$. TNT-concentrations were calculated using analytical standards (AccuStandard Explosives Inc., New Haven, USA). The concentration of explosives in the contaminated soil was on average $48,835 \mathrm{mg}$ TNT per $\mathrm{kg}$ DW soil, $190 \mathrm{mg}$ 1,3,5-trinitrobenzene, $73 \mathrm{mg}$ nitrobenzene, 1,267 mg aminodinitrotoluene, and $182 \mathrm{mg}$ dinitrotoluene per $\mathrm{kg} \mathrm{DW}$ soil. No TNT or related nitroaromatics were detected in the noncontaminated bulk soil or rhizosphere $(<0.01 \mathrm{ppm}$ detection limit).

One hundred gram soil samples were collected from the top $5-10 \mathrm{~cm}$ after manually removing the fallen debris and litter. Three replicate soil samples were collected within $20 \mathrm{~cm}$ and pooled together to make one sample. Rhizosphere samples were collected from the roots of A. pseudoplatanus trees according to standard procedures. All samples were kept at $4^{\circ} \mathrm{C}$ during transport to laboratory. In the laboratory, bulk soil was sieved using a two mm sieve to homogenize the samples. Rhizosphere soil was obtained by shaking and vigorously vortexing the roots (10 min at max. speed) in sterile P-buffer (per l: $2.36 \mathrm{~g} \mathrm{Na}_{2} \mathrm{HPO}_{4}$; $1.80 \mathrm{~g} \mathrm{NaH}_{2} \mathrm{PO}_{4}, 85.0 \mathrm{~g} \mathrm{NaCl}$ and $200 \mu \mathrm{l}$ Tween 80; $\mathrm{pH}$ 6.8). The tubes were subjected to centrifugation (2,500 g, $20 \mathrm{~min}$ ), and the resulting pellet was kept as the rhizosphere soil. All soil samples were flash-frozen in liquid nitrogen and stored at $-80^{\circ} \mathrm{C}$ until DNA was extracted.

\section{DNA Extraction, PCR Amplification, and Pyrosequencing}

Approximately $400 \mathrm{mg}$ of soil was used for each DNA extraction. DNA was extracted in triplicate from each pooled sample using a PowerSoil DNA Isolation Kit according to the manufacturer's protocol (MoBio, Carlsbad, CA, USA). This resulted in three replicates for each of six pooled soil samples. Subsequently, amplicon libraries were created using barcode-tagged primers for the primer pairs $68 \mathrm{f} / 518 \mathrm{r}$, 341f/785r, 799f/1193r, 967f/1391r generating amplicons of $\sim 400$ bp (Table 1). Both forward and reverse primers were synthesized with the Roche 454 pyrosequencing adaptors (Lib-L A and B) and sample-specific $10 \mathrm{bp}$ multiplex identifier (MID) to allow for sample binning after sequencing (Roche Applied Science, Mannheim, Germany). The forward fusion primer design was $5^{\prime}$ CCATCTCATCCCTGCGTGTCTCCGACTCAG NNNNNNNNNN-forward primer $3^{\prime}$, and the reverse primer design was $5^{\prime}$ CCTATCCCCTGTGTGCCTTGGCAGTCTCAGreverse primer $3^{\prime}$. A two-round amplification process was used to amplify the DNA samples to reduce dimer formation that is more frequent when using long fusion primers directly on complex genomic DNA-templates. In addition, the first PCR produces homogenous sized amplicons resulting in a more efficient second PCR using the fusion primers (Wu et al., 2010; Berry et al., 2011). The DNA-samples were amplified using a Techne TC5000 thermocycler (Bibby Scientific Limited, Staffordshire, UK) under the following conditions: initial denaturation at $95^{\circ} \mathrm{C}$ for $2 \mathrm{~min}$, followed by 25 cycles ( $1 \mathrm{st}$ PCR) or 10 cycles (2nd PCR) of denaturation at $95^{\circ} \mathrm{C}$ for $30 \mathrm{~s}$, annealing at $53^{\circ} \mathrm{C}$ for $40 \mathrm{~s}$ and extension at $72^{\circ} \mathrm{C}$ for $1 \mathrm{~min}$, with a final extension performed at $72^{\circ} \mathrm{C}$ for $10 \mathrm{~min}$. Reactions were carried out in $25 \mu \mathrm{l}$ reaction volumes using the FastStart High Fidelity PCR System (Roche Applied Science, Mannheim, Germany). Each reaction contained $2.75 \mathrm{ml}$ FastStart $10 \times$ reaction buffer, $1.8 \mathrm{mM} \mathrm{MgCl}_{2}, 0.2 \mathrm{mM}$ dNTP mix, $0.3 \mathrm{mM}$ of each primer, $1.25 \mathrm{U}$ FastStart HiFi polymerase and $5 \mathrm{ng}$ of template DNA for the first round of PCR (as measured using a Nanodrop spectrophotometer). Amplified DNA from the first PCR was purified from 1.5\% agarose gel using a QIAquick gel extraction kit (Qiagen, Venlo, Netherlands), and $1 \mu \mathrm{l}$ of the purified PCRproducts was used for the second PCR with the MID-tagged fusion primers using 10 PCR cycles instead of 25. The amplified DNA was cleared from PCR primers and primer dimers using the QIAquick PCR purification kit (Qiagen, Venlo, Netherlands). Finally, purified DNA was quantitated using the Quant-iT

TABLE 1 | Primers used in the current study.

\begin{tabular}{|c|c|c|c|c|c|c|c|c|}
\hline Primer pair $^{\mathbf{a}}$ & Sequence $\left(5^{\prime}-3^{\prime}\right)$ & Position in E. coli ${ }^{\mathrm{a}}$ & V-regions & Amplicon size & \multicolumn{3}{|c|}{ Domain coverage $(\%)^{\mathbf{b}}$} & References \\
\hline $518 r$ & WTTACCGCGGCTGCTGG & $518-534$ & & & & & & Lee et al., 2010 \\
\hline $341 f$ & CCTACGGGNGGCWGCAG & $341-357$ & V3-V4 & 444 & 96.51 & 82.96 & 0.14 & Klindworth et al., 2013 \\
\hline $785 r$ & GACTACHVGGGTATCTAATCC & $785-805$ & & & & & & \\
\hline $967 f$ & CAACGCGAAGAACCTTACC & $967-985$ & V6-V8 & 424 & 66.54 & 0.007 & 0.03 & Sogin et al., 2006 \\
\hline $1391 r$ & GACGGGCGGTGWGTRCA & $1391-1407$ & & & & & & Walker and Pace, 2007 \\
\hline
\end{tabular}

Primer sequence, 165 rRNA gene variable region, amplicon size, domain coverage rate and literature reference.

a Primer position according to E. coli numbering (Baker et al., 2003) umulative-sum-scal.

${ }^{b}$ Primer pair coverage was calculated using PrimerProspector 1.0.1 according to the non-redundant SILVA SSU r123 database and the weighted score method (Walters et al., 2011$)$. 
PicoGreen dsDNA assay kit (Life Technologies Europe, Gent, Belgium) and a Fluostar Omega plate reader (BMG Labtech, Ortenberg, Germany) prior to pooling amplicons in equimolar concentrations in $100 \mu \mathrm{l}$ TE buffer. Correct amplicon size and integrity were checked by analysing $1 \mu l$ of the library on a DNAchip (DNA 1000 kit, Agilent Technologies, Diegem, Belgium) on a 2100 Bioanalyser (Agilent Mannheim, Germany) according to the manufacturer's instructions. The resulting quality checked amplicon pool, containing all 24 samples were sequenced using a Roche Genome Sequencer FLX System at LGC Genomics (Berlin, Germany).

\section{Bioinformatics Processing}

The raw sequence data (SFF-files) were pre-processed in QIIME v1.9.1 (Caporaso et al., 2010b) using a custom pipeline and the Flemish Supercomputer Centre (VSC). Sequences were trimmed to a length of $400 \mathrm{bp}$ and were only included in the analyses that matched perfectly to the barcode, had $<6$ homopolymers, no ambiguous bases, a minimal quality score of 25 over a window of $50 \mathrm{bp}$, and $<2$ mismatches in the primers (in case of degenerate primers). Next, the sequences were checked for chimeras using UCHIME v4.2.40 (Edgar et al., 2011) with usearch61_minh set to 1 to reduce the number of false-positive chimeras. After removing the chimeric sequences, the sequences that passed preprocessing were clustered in operational taxonomic units (OTUs) at 97\% similarity using Uclust v1.2.22 and closed-reference OTUpicking in QIIME (Caporaso et al., 2010b). Taxonomy was assigned using the SILVA v123 database (Quast et al., 2013). We have chosen SILVA as we wanted to confirm that the primer pairs did not amplify eukaryotes, which are represented in the SILVA database and not in the Greengenes databases. The OTU-table was subsequently depleted of non-target sequences (plant-organelles, Eukaryota, Archaea). The final representative sequence set was aligned using PYNAST v0.1 (Caporaso et al., 2010a) and the core alignment of the SILVA database release 123. Finally, alignments were filtered using the PH Lane mask (Caporaso et al., 2010a) and phylogenetic trees were constructed from the filtered alignments using FastTree v2.1.3 (Price et al., 2009).

Rarefaction curves were constructed for each sample individually as well as per primer pair and soil type using QIIME (Caporaso et al., 2010b). Intra-sample diversity measures including phylogenetic richness, observed species, Shannon diversity and Inverse Simpson were calculated in QIIME on OTU tables that were subsampled to 1,000 reads without replacement as in the hypergeometric model (Colwell et al., 2012) and on non-normalized OTU-tables. Taxonomic plots of the relative abundance data were visualized using Explicet v2.10.5 (Robertson et al., 2013). MetagenomeSeq's cumulative-sum-scaling (CSS; Paulson et al., 2013) was used as normalization method prior to $\beta$-diversity analyses. Additionally, global singletons were filtered from the OTU-tables using the filter_otus_from_otu_table script in QIIME. Principalcoordinate analysis (PCoA) was performed on the weighted UniFrac matrices (Lozupone and Knight, 2007) to assess the clustering patterns observed for the samples amplified with the different primers. Permutational multivariate analysis was used to identify whether the observed clustering was significant based on the ANOSIM function in QIIME (Caporaso et al., 2010b). Hierarchical trees were generated from the weighted UniFrac distance matrix and Bray-Curtis dissimilarity matrix using Unweighted Pair Group Method with Arithmetic Mean (UPGMA; Felsenstein, 2003) and Jackknife tree node support.

\section{Differential Abundance Testing}

To identify differently abundant OTUs between the primer sets or between the soil types, non-parametric and parametric tests were employed. For the non-parametric test, Linear Discriminant Analysis with Effect Size (LEfSe) was used on the relative abundance OTU table (Segata et al., 2011). In brief, KruskalWallis (KW) sum-rank test was run at $p<0.05$ and in the second step, the Wilcoxon rank-sum test was run at $p<0.05$. Then Linear Discriminant Analysis (LDA) was run at different effect sizes to detect the most important differently abundant clades. The software package STAMP 8.30 was also used to detect differentially abundant OTUs between two sample groups using the Welch's $t$-test at $p<0.05$ with 10,000 bootstraps (Parks et al., 2014). For the parametric approach, the zero-inflated Gaussian model (fitZIG) was fitted using the package MetagenomeSeq in R 3.3.0 (Paulson et al., 2013). From the normalized data, a dual heatmap-dendrogram of significant OTUs was constructed based on the $\log 2$-transformed abundance values and BrayCurtis clustering algorithm.

Other statistical analyses were performed in $\mathrm{R}$ version 3.3.0 (The R Foundation for Statistical Computing, Vienna, Austria), Past 3.x (http://folk.uio.no/ohammer/past/), and Sigmaplot 11.0 (Systat Software, California, USA). Normal distributions of the residuals of models were checked with the Shapiro-Wilk test, while homoscedasticity of variances was analyzed using the Bartlett's test. Depending on the distribution of the estimated parameters, either ANOVA or the Kruskal-Wallis Rank Sum Test was used to check for significant differences in variances of parameters. Pearson rank sum correlations were run on the biological replicates using Sigmaplot 11.0. Graphical tree representations of differentially expressed clades were generated using GraPhIan (http://huttenhower.sph.harvard.edu/graphlan). To visualize the shared and unique OTUs between samples, the tool Venny 2.1.0 was used (Oliveros, 2007).

\section{Quantitative Real-Time PCR}

To test the primer pair amplification efficiency on heterogeneous environmental DNA, a two-fold dilution series (1:1-1:32) was made from 12 DNA samples (ranging from 5 to $0.15 \mathrm{ng}$ per $\mu \mathrm{l}$ ), and amplified with qPCR. Amplification was performed in optical 96 well plates using a 7500 Fast Real-Time PCR System (Applied Biosystems, Foster City, CA, USA) and SYBR Green chemistry. PCR conditions were as follows: initial denaturation at $95^{\circ} \mathrm{C}$ for $2 \mathrm{~min}$, followed by 40 cycles of $95^{\circ} \mathrm{C}(30 \mathrm{~s}), 53^{\circ} \mathrm{C}$ $(30 \mathrm{~s})$, and $72^{\circ} \mathrm{C}(60 \mathrm{~s})$ and a final extension phase at $72^{\circ} \mathrm{C}$ for $30 \mathrm{~s}$ followed by the generation of a dissociation curve to verify amplification specificity. Reactions contained $2.5 \mu \mathrm{L}$ template DNA, $5 \mu \mathrm{L}$ 2x Fast SYBR Green Master Mix (Applied Biosystems, Foster City, CA, USA), $0.3 \mu \mathrm{l}$ forward and reverse primers $(0.3$ $\mathrm{mM}$ final concentration) and $1.9 \mu \mathrm{L}$ nuclease-free $\mathrm{H}_{2} \mathrm{O}$ in a total 
volume of $10 \mu \mathrm{L}$. PCR efficiencies (E) were calculated as $\mathrm{E}=$ $\left(10^{-1 / \text { slope }}-1\right) \times 100$.

To evaluate a potential PCR-bias for individual strains, DNA was extracted from 13 pure cultures isolated previously from the TNT-contaminated soil, including Achromobacter sp. zw108, Arthrobacter sp. zw99, Bacillus licheniformis zw124, Chryseobacterium sp. zw156, Herbaspirillum sp. zw98, Nocardia sp. zw144, Paenibacillus sp. zw125, Pseudomonas sp. zw136, Rhizobium sp. zw143, Rhodococcus sp. zw143, Sphingobium sp. zw115 and Variovorax sp. zw103. DNA was extracted from cultures using the Qiagen Blood and Tissue Kit according to the manufacturer's instructions (Qiagen, Venlo, Netherlands).

To compare the 454-sequence data with qPCR, the same soil samples were amplified using Bacteria domain and group specific qPCR primers (Supplementary Table 1). The log copy number per ng DNA and relative abundances were calculated using standard curves of plasmids. These plasmid-standards were prepared from Escherichia coli, Okibacterium fritillariae (phylum Actinobacteria), Chryseobacterium vrystaatense (phylum Bacteroidetes), Bacillus mycoides (phylum Firmicutes), Novosphingobium barchaimii (class Alphaproteobacteria), and Burkholderia sediminicola (class Betaproteobacteria). In brief, 16S rRNA gene amplicons of these strains were obtained by PCR amplification with the selected primers and cloned in the pGEM-T Vector (Promega Benelux, Leiden, the Netherlands). The vectors were incorporated in competent E. coli JM109 cells (Promega Benelux, Leiden, the Netherlands). Plasmids were isolated from successfully transformed cells using the UltraClean Standard Mini Plasmid Prep Kit (MO BIO Laboratories, Carlsbad, California, USA) and sent for Sanger sequencing (Macrogen Europe, Amsterdam, The Netherlands) to verify insert sequence. DNA concentrations of the plasmid suspensions were determined using Quant-iT PicoGreen (Life Technologies, Carlsbad, California, USA). Ten-fold serial dilutions of the plasmid suspensions (ranging from $10^{1}$ to $10^{8}$ copies per $\mu \mathrm{l}$ ) were made in triplicate for all taxa including two no-template controls. PCR conditions used were: initial denaturation at $95^{\circ} \mathrm{C}$ for $15 \mathrm{~min}, 40$ cycles of $94^{\circ} \mathrm{C}$ for $15 \mathrm{~s}, 30 \mathrm{~s}$ at the annealing temperature, $72^{\circ} \mathrm{C}$ for $30 \mathrm{~s}$. Annealing temperatures were experimentally optimized to maximize the specificity of amplification (Supplementary Table 1). Each reaction was set up in triplicate. Reactions contained $2 \mu \mathrm{L}$ DNA template, 5 $\mu \mathrm{L}$ of $2 \mathrm{x}$ QuantiTect SYBR Green PCR Master Mix (Qiagen, Venlo, Netherlands), $0.2-0.8 \mu \mathrm{L}$ of each primer [100-400 nM; depending on taxon (Supplementary Table 1)], and nuclease-free $\mathrm{H}_{2} \mathrm{O}$ in a total volume of $10 \mu \mathrm{L}$. Amplification was linear $\left(R^{2}\right.$ $>0.99$ ) over six orders of dynamic range from $10^{3}$ to $10^{8}$ copies per $\mu \mathrm{L}$, and efficiencies reached between 81 and $93.8 \%$ (Supplementary Figure 1). Melting curves were evaluated to confirm that the detected fluorescence originated from specific products and not from primer dimers or other amplification artifacts.

\section{In silico Primer Evaluation}

To evaluate the primer-to-target mismatches in silico, primers were tested with PrimerProspector 1.0.1 (Walters et al., 2011) against the SILVA SSU ref NR database version 123 containing 597,607 16S/18S sequences (https://www.arb-silva.de/). All tests were performed as described by Walters et al. (2011) using standard settings. Primer scores were calculated based on the following formula: weighted score $=$ non $-3^{\prime}$ mismatches $\times 0.40$ $+3^{\prime}$ mismatches $\times 1.00+$ non $-3^{\prime}$ gaps $\times 1.00+3^{\prime}$ gaps $\times 3.00$. An additional penalty score of 3.00 was assigned if the final $3^{\prime}$ base of a primer had a mismatch with its target sequence (Walters et al., 2011). The closer the PrimerProspector value is to 0 , the better the primers perform theoretically.

\section{Accession Numbers}

The standard flowgram format (SFF) files were deposited in the European Nucleotide Archive under study accession PRJEB18468 and sample accession numbers ERS1463796ERS1463819.

\section{RESULTS}

\section{Read Output and Sequence Length Distribution of 454 Amplicon Pyrosequencing Data}

The 454 amplicon sequencing run generated a total of 116,739 raw reads with average raw read lengths of $420 \mathrm{bp}$ for the primers tested. After read processing and quality filtering in QIIME, a total of 71,821 high quality bacterial reads were retained (Supplementary Table 2). The primer pairs 68f/518r, 341f/785r and 799f/1193r yielded an average of 14,442 sequences, and the dataset amplified with $967 \mathrm{f} / 1391 \mathrm{r}$ had a total of 28,498 reads. The difference in read number was not related to difference in primer pair performance, as the amplicons were sequenced together with those of another unrelated study. The primers were found to be specific for Bacteria as almost no Eukaryotic or Archaeal sequences were amplified. Though some primer pairs were more likely to amplify chloroplast and mitochondrial reads, with 5,910 chloroplast reads for $68 \mathrm{f}-518 \mathrm{r}$ being the highest, and only 1 for 799f/1193r.

From the high quality read sequence length distribution graph it can be observed that primers 68f/518r and 341f/785r produced additional peaks next to the peak of the expected size, compared to primers 799f/1193r and 967f/1391r (Supplementary Figure 2A). The shorter amplicons with 490 bp length produced by $341 \mathrm{f} / 785$ were almost exclusively assigned to Alphaproteobacteria as shown by a blast search of 1,000 randomly selected sequences (Supplementary Figure 2A). Similarly, the peak of small amplicons produced by primer pair $68 \mathrm{f} / 518 \mathrm{r}$ (410 bp) was dominated by reads assigned to Alphaproteobacteria, while the 440 and 460 bp sequences represented a more diverse set of bacterial taxa (Supplementary Figures 2A, 3). Examination of sequence length distribution showed that $98 \%$ of the shortest reads (high peak at $410 \mathrm{bp}$ ) were assigned to Rhizobiales, of which $89 \%$ were assigned to Bradyrhizobia, and 49\% uncultured Bradyrhizobium sequences.

\section{Parametric Analyses of Pyrosequencing Data}

The 71,821 bacterial sequences that clustered at $97 \%$ identity threshold generated a total of 8,169 OTUs using the closed reference OTU picking strategy. Rarefaction curves for the 
total number of observed OTUs and phylogenetic diversity demonstrated that primer pair 341f/785r generated the steepest slope and highest diversity, followed by 967f/1391r, 799f/1193r, and 68f/518r (Figures 1A,B). The observed number of OTUs and Faiths PD index was significantly higher for 341f/785r compared to the other primer pairs (Kruskal-Wallis, $p<$ 0.05) based on calculations with normalized reads (Figure 2) and non-normalized reads (Supplementary Figure 3). In detail, primer pair 341f/785r detected on average $599 \pm 4.8$ OTUs and the phylogenetic richness was $43 \pm 0.3$. While there was no significant difference in the number of observed species detected by primer pair $967 \mathrm{r} / 1391 \mathrm{r}(493 \pm 8.4)$ and $799 \mathrm{f} / 1193 \mathrm{r}(401 \pm$ $10)$, primer pair $68 \mathrm{f} / 518 \mathrm{r}$ detected a significantly lower number $(214 \pm 5$; Figure 2; Kruskal-Wallis, $p<0.05)$. The same trend was observed for the Chao richness, with the statistically highest values being for $341 \mathrm{f} / 785 \mathrm{r}(1781 \pm 183)$, followed by $967 \mathrm{f} / 1391 \mathrm{r}$ $(1306 \pm 334), 799 \mathrm{f} / 1193 \mathrm{r}(1112 \pm 298)$, and 68f/518r (557 \pm 103 ; Kruskal-Wallis, $p<0.05)$.

The rarefaction curves for the Shannon diversity and Inverse Simpson index revealed the same trends as observed for OTU richness and PD diversity (Supplementary Figures 4, 5). The Shannon diversity index value showed that primer pair $341 \mathrm{f} / 785 \mathrm{r}$ had the highest diversity based on the most abundant bacteria, ranging from 8.4 to 8.9 , while $68 \mathrm{f} / 518 \mathrm{r}$ had the lowest, ranging from 3.2 to 5.4, and these differences were significant between the primer pairs $(p<0.05)$. Primer pairs 799f/1193r and 967f/1391r performed second best with similar Shannon indices, average of $7.76 \pm 0.06$. The inverse Simpson index showed the same trend as observed for the Shannon index.

Besides the alpha-diversity differences between the primer pairs, the data also revealed differences between the soil samples tested in the observed number of OTUs, Faith's PD, and diversity (Figures 1C,D). The non-contaminated bulk soil samples were more diverse than the TNT-contaminated bulk soil in terms of Shannon diversity and Inverse Simpson, as demonstrated by all primer pairs (Supplementary Table 3). Further, there were also primer pair dependent differences in taxon richness. Primer pair 341f/785r detected a significant lower taxon richness in the TNT-contaminated bulk soil samples $(565 \pm 12)$ compared to the non-contaminated bulk soil $(622 \pm 5)$ and rhizosphere samples $(641 \pm 14)$, while the other primer pairs detected only a decreasing trend (Supplementary Table 3). On the other hand, primer pair 967f/1391r detected a significantly higher number of OTUs in the rhizosphere ( $456 \pm 4$ ) compared to the bulk soil $(427 \pm 17)$, while no statistical difference was observed for primer pairs 341f/785r and 799f/1193r, and a significant decrease with 68f/518r.
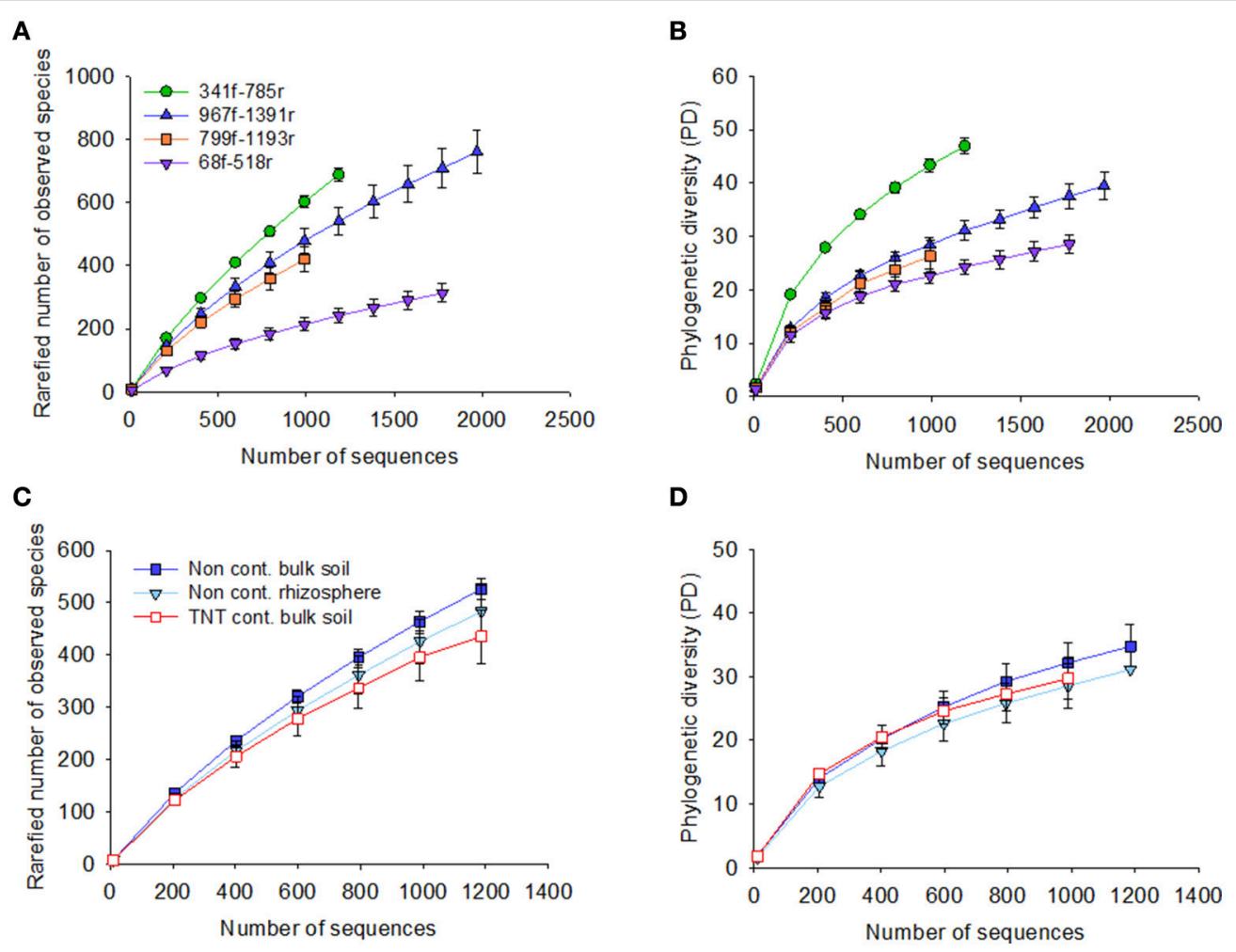

D

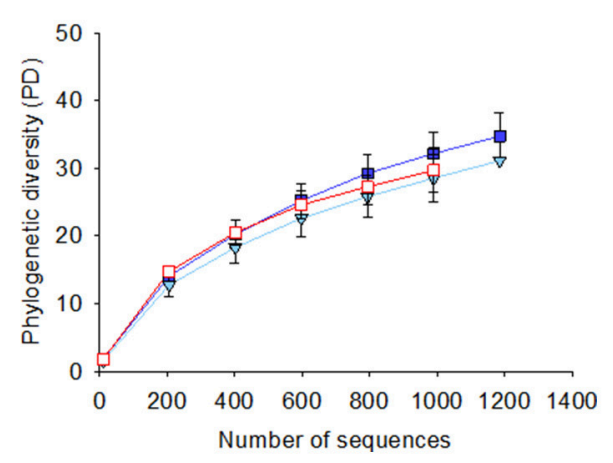

FIGURE 1 | Rarefaction curves for the four primer pairs used in this study, 68f/518r, 341f/785r, 799f/1193r, 967f/1391r. Graphs show the rarefaction curves of 16S rRNA gene sequences based on pyrosequencing of the bulk soil and Acer pseudoplatanus rhizosphere soil samples collected from a military forest, Zwijndrecht, Belgium. The number of sequences were plotted against the rarefied number of observed species based on $97 \%$ sequence similarity cut-off value, and phylogenetic diversity for each of the primer pairs (A,B, respectively), and per soil type (C,D). 


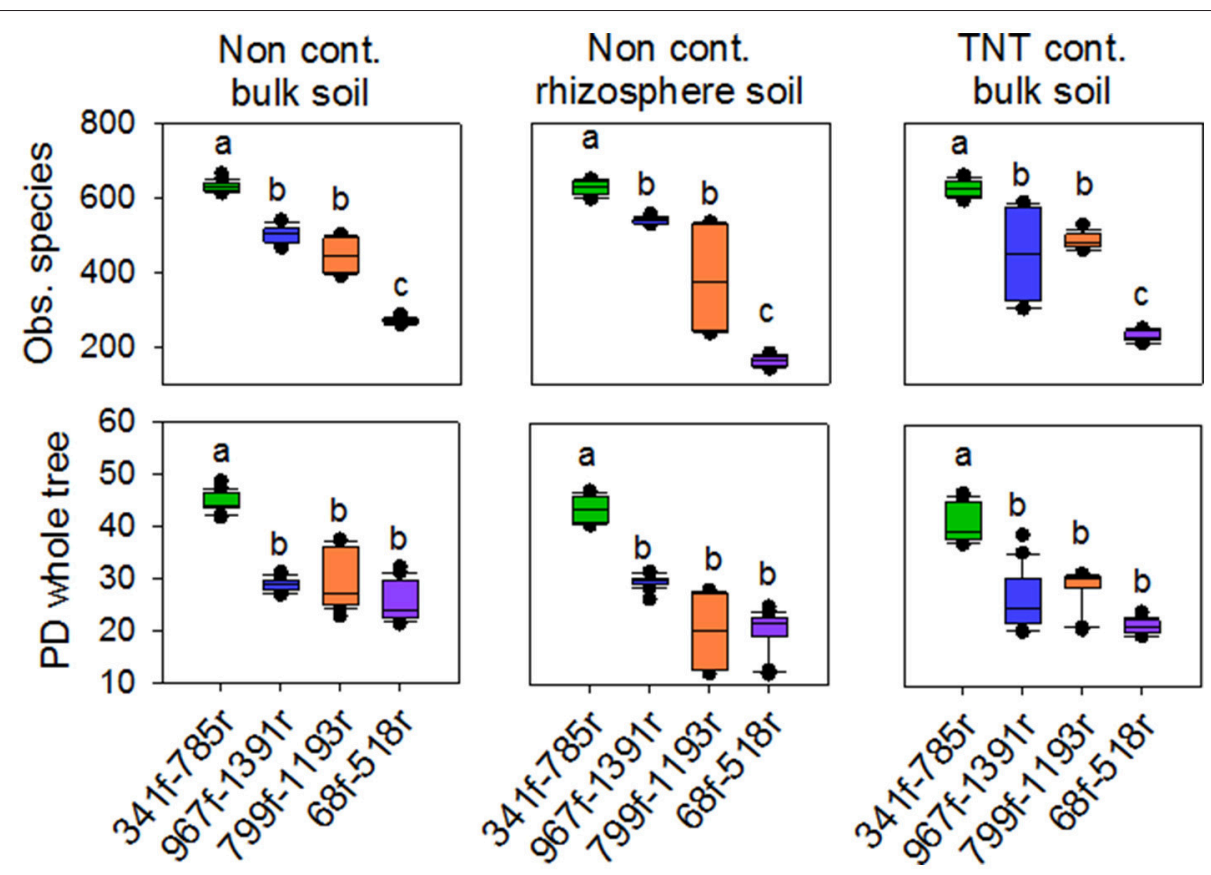

FIGURE 2 | Box plots of observed species and phylogenetic diversity for the four primer pairs used in this study, 68f/518r, 341f/785r, 799f/1193r, 967f/1391r. Average number of observed species was based on $97 \%$ sequence similarity cut-off value. Averages were calculated based on a rarefied depth of 1,000 sequences per sample, across the replicates for each primer pair. Different letters denote significant differences (Kruskal Wallis, $p<0.05$ ).

\section{Phylum-Level Taxonomic Distribution}

All four primer pairs detected a different number of phyla and class level taxa. A total of 19 phyla were detected by $68 \mathrm{f} / 518 \mathrm{r}, 27$ by $341 \mathrm{f} / 785 \mathrm{r}, 25$ by $799 \mathrm{f} / 1191 \mathrm{r}$, and 28 by $967 \mathrm{f} / 1391 \mathrm{r}$ (Figure 3A, Supplementary Table 4). The twelve most abundant phyla/classes that were detected by all primer pairs included Alphaproteobacteria, Bacteroidetes, Gammaproteobacteria, Betaproteobacteria, Acidobacteria, Actinobacteria, Chloroflexi, Planctomycetes, Deltaproteobacteria, Nitrospirae, Verrucomicrobia, and Firmicutes (Figure 3A). Some of the more abundant phyla not detected by primer pair 68f/518r in comparison to the other primer pairs were Chlamydiae, Chlorobi, and Gemmatimonadetes (Supplementary Table 4). Examples of primer pair differences in many lower abundance groups can be observed in Supplementary Table 4.

The relative abundances of the major phyla were also significantly different (Figure 3A). Primer pair 68f/518r was significantly biased toward Alphaproteobacteria $(60-80 \%$ of the sequences, LDA-score $>4$, LefSe) compared to the other primer pairs where the communities were more evenly distributed (Figure 3A). A statistically higher relative abundance of Bacteroidetes and Actinobacteria was detected by $967 \mathrm{f} / 1391 \mathrm{r}$ compared to the other primer pairs (LDA-score $>4$, LefSe $p$ $<0.05$ ), while Betaproteobacteria sequences were significantly more amplified by primer 799f/1193r (LDA-score > 4, LefSe $p<0.05$ ). Interestingly, a comparison of the shared and single OTUs showed that only a small percentage of the OTUs were shared among all 4 primer pairs $(0.5 \%$; Supplementary Figure 6). Combining the data from 341f/785r,
$799 f / 1193 r$, and $967 f / 1391 \mathrm{r}$ however covered over $90 \%$ of the OTUs.

Results of the weighted UniFrac distance PCoA plot demonstrated significant clustering of the samples according to the primer pair used rather than soil type (ANOSIM R $=0.82$, $p<0.001$; Figure 3C). Bray-Curtis cluster analyses of the 150 most dominant OTUs showed a separate grouping for $68 \mathrm{f} / 518 \mathrm{r}$, but the other primer pairs clustered in 1 group rather than 3 separate. Besides primer $68 \mathrm{f} / 518 \mathrm{r}$, the heatmap also showed that the TNT-contaminated soil samples clustered in a separate group from the non-contaminated bulk soil and rhizosphere samples with $75 \%$ Jackknife support, while the non-contaminated bulk soil and rhizosphere communities could not be significantly discriminated from each other (NPMANOVA, $p<0.26$ ).

\section{Family, and Genus-Level PCR Bias}

The relative abundance of the 10 most abundant families detected differed between the primer pairs (Figure 3B). In total, 32 bacterial groups were distinct to at least one primer pair using the logarithmic (LDA) value of 4 , and test-values of $p<0.05$ (Supplementary Figure 7). Specifically, for primer pair $68 \mathrm{f} / 518 \mathrm{r}$, a bias was detected toward Bradyrhizobiaceae, Bradyrhizobium, and Rhizobiales (LDA score 5, LefSe at $p<$ 0.05) within the Alphaproteobacteria, compared to the other primer sets. For primer pair 967f/1391r, Chitinophagaceae and Sphingobacteriales were detected more often, followed by Terrimonas, Burkholderiales, Actinobacteria, Micrococcales, and others (Supplementary Figure 7), while Flavobacteriaceae, and Sphingomonadaceae were detected more often with primer pair 
A

Top 12 phyla/classes

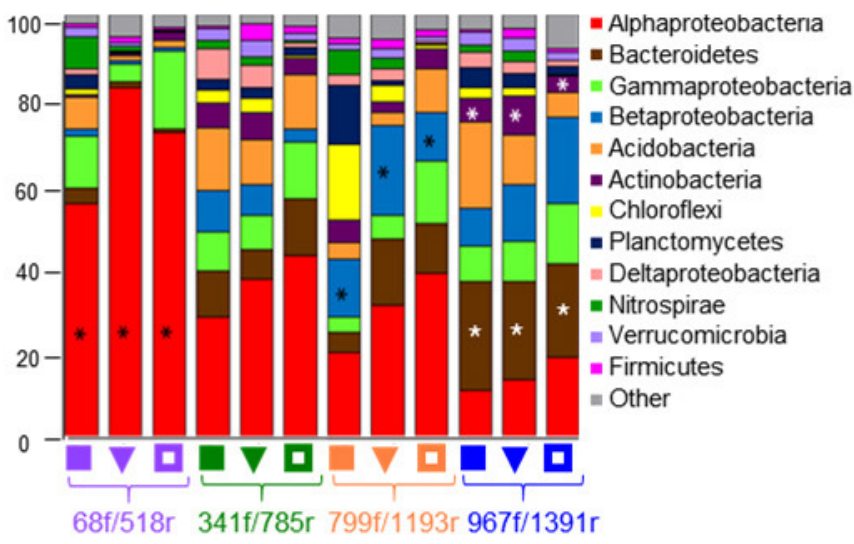

B

\section{Top 7 families}
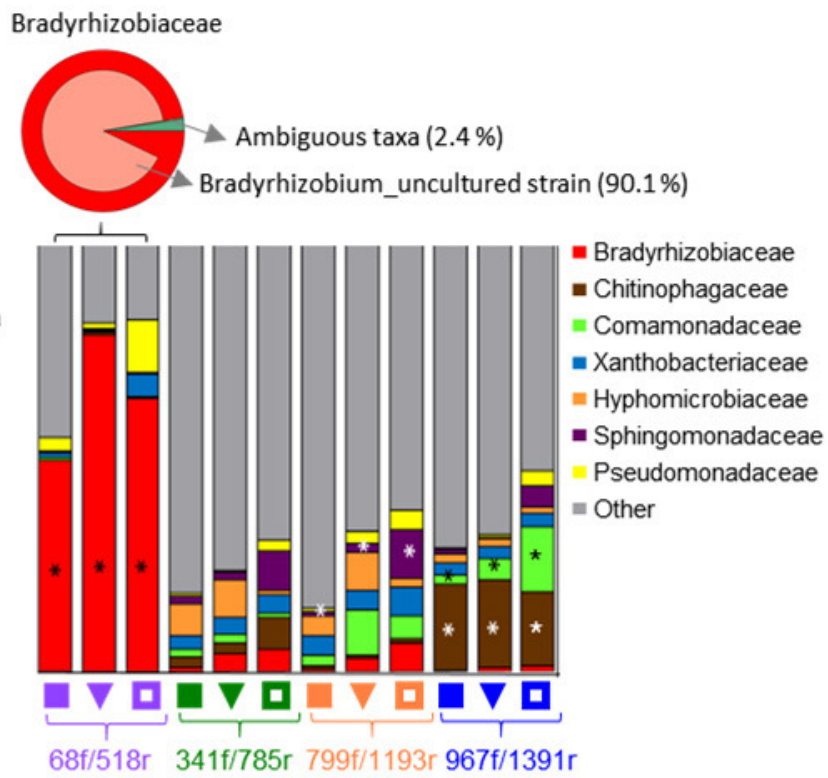

C

Principal Coordinate Analysis of weighted UniFrac distance

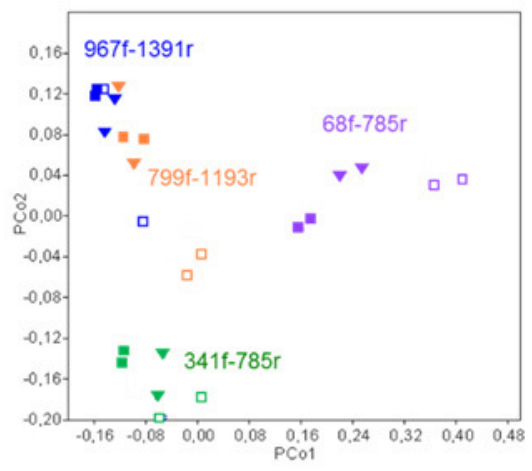

D

Heatmap of log2 OTU abundance Clustering by Bray-Curtis dissimilarity Based on fitZIG normalised OTU-table

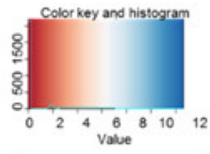

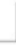

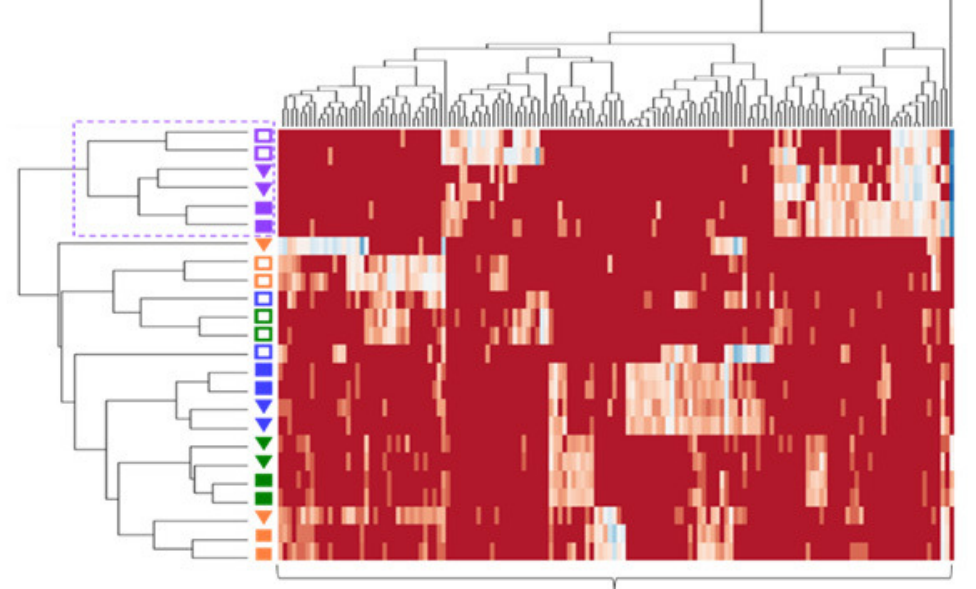

150 OTUs with the largest overall variance

\section{$799 f / 1193 r$}

Non cont. bulk soil

TNT cont. bulk soil

Non cont. mizosphere soil

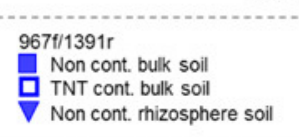

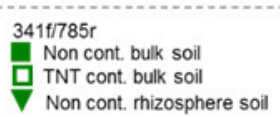

FIGURE 3 | Abundance distribution of the most dominant phyla, Proteobacteria classes, and families, principal coordinate analyses, and heatmap of the top 150 OTUs. The relative abundance bar charts show the 12 most dominant phyla and Proteobacteria classes (A) and seven most dominant families (B) per primer pair and soil type, based on pyrosequencing data of bulk soil and Acer pseudoplatanus rhizosphere soil samples, Zwijndrecht, Belgium. Significant different lineages between the primers are indicated with a star (Kruskal-Wallis $p<0.05$ ). (C) PcoA was calculated based on the CSS-normalized data and weigthed UniFrac distance matrix in QIIME. (D) The heatmap was generated based on the fitZIG normalized OTU-table (Paulson et al., 2013) and shows the 150 OTUs with largest overall variance clustered by Bray-Curtis dissimilarity. 
799f/1193r. The fine-level bacterial groups that showed an LDA value higher than 4 with $341 \mathrm{f} / 785 \mathrm{r}$ included Caulobacterales, Hyphomicrobiaceae, and Rhodospirillales.

Between soil samples, differences in the distribution of bacterial families and finer-level lineage were observed depending on the primer pairs used. Commonly for all primer pairs, 59 bacterial features (from phylum to species level) were found to differ between the uncontaminated bulk soil, TNTcontaminated bulk soil, and non-contaminated rhizosphere samples at an LDA of $2, p<0.05$. Supplementary Figure 8 shows the most significant features with LDA scores of 3.5 or higher, with Acidobacteriaceae/subgroup3, Acidobacteriales, Gammaproteobacteria, Mizugakiibacter, and Rhodanobacter enriched in the TNT-contaminated bulk soil, whereas Nitrospira, Pedomicrobium, and many uncultured bacteria belonging to other Acidobacterial groups were significantly enriched in the non-contaminated bulk soil, and Clostridia, Clostridiales, and Firmicutes were more enriched in the rhizosphere soils. We also noted the significantly enriched clades in the TNT-contaminated bulk soil for primer pair 341f/785r using Welch's $t$-test $(p<0.05)$ in STAMP (Supplementary Figure 9). This revealed an enrichment in the TNTpolluted soil of Xanthomonadaceae (Gammaproteobacteria), Bradyrhizobiaceae, Caulobacteracae, the Incertae Sedis group of the Rhizobiales (Alphaproteobacteria), and Chitinophagaceae (Bacteroidetes) amongst others, while several subgroups of the Acidobacteria, Deltaproteobacteria, and Betaproteobacteria were significantly depleted (Welch's $t$-test, $p<0.05$; Supplementary Figure 9).

\section{Primer Pair Efficiency}

The average PCR efficiencies ( \pm standard error) were not statistically different between the primer pairs, and were calculated to be $96 \%( \pm 1 \%)$ for $341 \mathrm{f} / 785 \mathrm{r}, 105 \%( \pm 2 \%)$ for $799 \mathrm{f} / 1193 \mathrm{r}, 101 \%( \pm 0.2 \%)$ for $68 \mathrm{f} / 518 \mathrm{r}$, and $99 \%( \pm 0.6 \%)$ for 967f/1391r. Additionally, gel electrophoresis and dissociation curves were used to confirm the presence of amplicons of the expected size. Exponential amplification was obtained between 19 and 21 PCR cycles for all soil samples and primer pairs, and the dynamic range for the primer pairs spanned from 5 $\mathrm{ng} / \mu \mathrm{l}$ to $50 \mathrm{pg} / \mu \mathrm{l}$. The linear regression of log-concentration versus $C q$ values for the four primer pairs showed Spearman $\mathrm{R}^{2}$ values from 0.95 to 0.99 . Similarly, no significant differences were found in amplification efficiency or sensitivity of the reference strains (Supplementary Figure 10). In some cases, primer pairs 967f/1391r and 341f/785r showed lower Cq values at exponential amplification (average of 17), compared to 21 for $68 \mathrm{f}-518 \mathrm{r}$ and 799f-1193r (average of 21), though not statistically significant.

\section{Reproducibility of Bacterial OTU Identification}

Scatterplots and Spearman Rank coefficients (Supplementary Figure 11) showed that the duplicate biological soil samples were most consistently identified by primer pair 68f/518r (average $\mathrm{r}$ of 0.25 ), followed by $341 \mathrm{f} / 785 \mathrm{r}$ ( $\mathrm{r}$ of 0.21 for the rhizosphere samples and 0.25 to 0.29 for the bulk soils). Primer pair $799 \mathrm{f} / 1193 \mathrm{r}$ showed reproducible identification of bacterial OTUs for the non-contaminated and TNT-contaminated bulk soil samples, but less for the rhizosphere samples ( $r$ 0.14), while primer pair 967f/1391r showed more variability between the biological replicates of TNT-contaminated samples (r 0.04).

\section{In silico Primer Evaluation}

The in silico analyses of the primer pairs against the latest SILVA r123 database showed that the PrimerProspector score was best for primer $341 \mathrm{f}(0.07 \pm 0.0005)$ and $785 \mathrm{r}(0.1 \pm 0.0006)$, while a good overall score $>0.5$ was obtained for $68 \mathrm{f}(0.83 \pm 0.002)$ and $1391 \mathrm{r}(0.875 \pm 0.002)$. The higher penalty score for $68 \mathrm{f}$ and 1391r was most likely caused by a higher occurrence of $3^{\prime}$ mismatches at the terminal base, namely 3.19 and $4.08 \%$ of the sequences, respectively. Primer 799f had also a higher percentage of sequences with $3^{\prime}$ end base mismatches (5.29\%), and this was due to the sequences from Cyanobacteria (mismatch with $96 \%$ of sequences) and Verrucomicrobia (mismatch with $48 \%$ of sequences; Supplementary Table 5). For 341f, $\sim 59 \%$ of Armatimonadetes sequences had a mismatch at the $3^{\prime}$ end terminus of the primer which can influence the amplification efficiency for this taxonomic group. For primer 68f, $40 \%$ of Chlorobi sequences showed a $3^{\prime}$ end mismatch. Overall, based on the average weighted PrimerProspector score and last base mismatches, the best primer pair would be $341 \mathrm{f} / 785 \mathrm{r}$, followed by $799 \mathrm{f} / 1193 \mathrm{r}, 68 \mathrm{f} / 518 \mathrm{r}$, and lastly $967 \mathrm{f} / 1391 \mathrm{r}$.

In silico taxonomic coverage of the primer pairs was predicted at the domain level (Table 1) and phylum level. The percentage non-coverage for the most abundant lineages (Figure 4) at the domain level showed that primer pair 341f/785r had the highest coverage for Bacteria representing $96.51 \%$ of the 159,615 bacterial sequences in the SILVA v123 database that were expected to be amplified (Table 1). The second best Bacterial domain coverage is expected from $799 \mathrm{f} / 1193 \mathrm{r}(85.55 \%)$, followed by $68 \mathrm{f} / 518 \mathrm{r}$ (78.59\%), and 967f/1391r (66.54\%). The coverage for the domains Archaea and Eukaryota was low $(<1.37 \%)$ for most primer pairs.

At the phylum level, it can be observed that primer pair $341 \mathrm{f} / 785 \mathrm{r}$ is theoretically able to amplify the most phyla, and this supports the findings from the experimental 454 pyrosequencing analyses (Figure 4). Depending on the scoring method used, total primer mismatch (tp_method) or average weighted score, different non-coverage rates were obtained (Figures $4 A, B$ ). In general, the tp_method in PrimerProspector has lower penalties, and tended to reflect better the sequencing results for the soil samples analyzed in this study. Using the tp_method, primer pairs 341f/785r and 799f/1193r showed the lowest noncoverage percentages (all $<10 \%$ ), while primer pairs $68 \mathrm{f} / 518 \mathrm{r}$ and $967 f / 1391$ had a non-coverage of $10 \%$ or higher for many phyla (Figure 4A). For example, primer pair 967f/1391r had a non-coverage percentage of $13.83 \%$ for Gammaproteobacteria, $28.3 \%$ for Gemmatimonadetes, and up to $81.25 \%$ for Chlorobi. Primer pair $68 \mathrm{f} / 518 \mathrm{r}$ had a non-coverage percentage of $25.17 \%$ for Armatimonadetes, $21.37 \%$ for Betaproteobacteria, $25.79 \%$ for Chloroflexi, 24.53\% for Firmicutes, 32.83\% for Saccharibacteria, and $24.71 \%$ for TA18. Other groups that are not covered by 68f/518r include Chrysiogenetes, Sbxy-6786, Cver-2, Oscillatoria, and MD2896-b258, amongst others (Supplementary Table 4). 
A

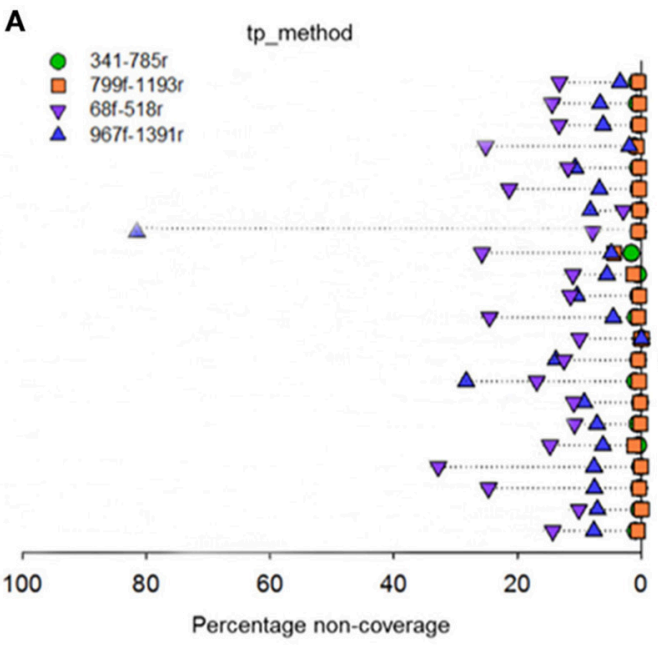

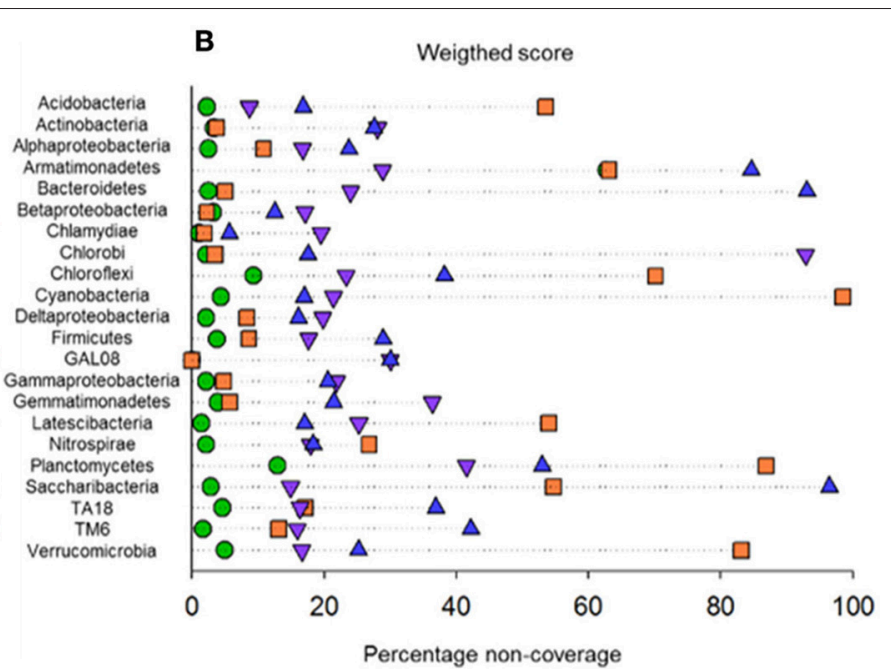

FIGURE 4 | Primer pair non-coverage percentage. Calculated by the total primer mismatch method (A) or using the weighted score method (B) in PrimerProspector.
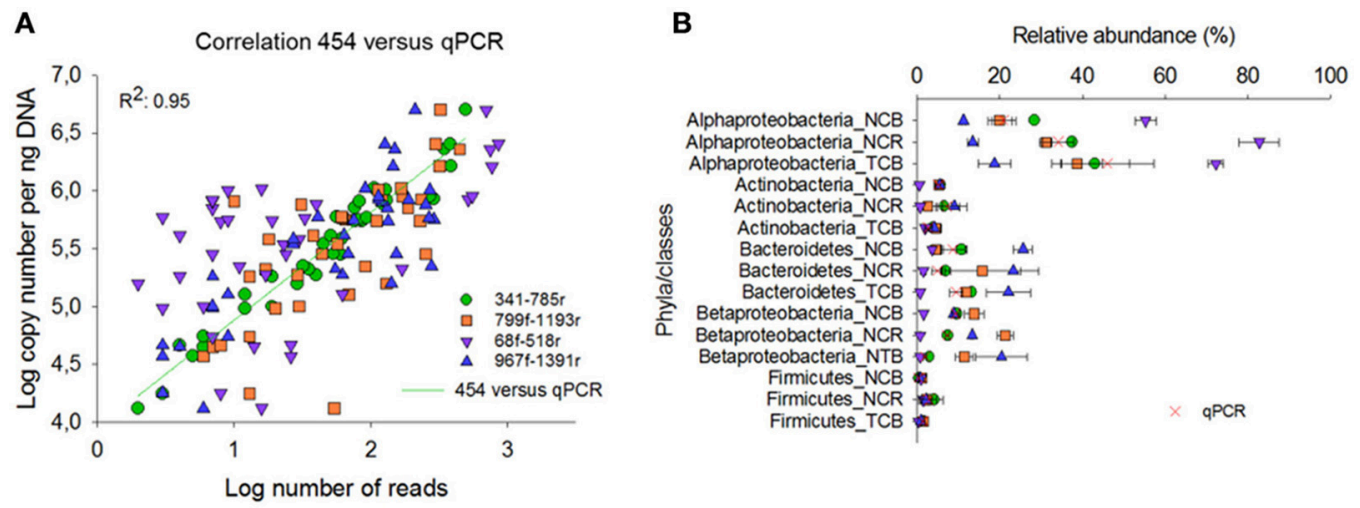

FIGURE 5 | Comparison of the relative abundance of different phyla and classes in the soil samples obtained by 454 pyrosequencing (A) and qPCR (B). NCB, non-contaminated bulk soil; NCR, non-contaminated rhizosphere soil; TCB, TNT-contaminated bulk soil.

\section{qPCR Analyses}

Compared with the qPCR results, primer pair 341f/785r showed the best correlation Spearman rank coefficient $(\mathrm{r}$ 97.5), followed by 967f-1391r (72.8\%), 799f-1193r (72.6\%), and $68 \mathrm{f}-518 \mathrm{r}$ (38.9\%; Figure 5A). The qPCR data were also expressed in relative percentage, by comparing phyla copy number to the copy number of total bacteria as estimated from the qPCR data of primer pairs EUB338f/EUB518r and Bact1369f/Prok1492r (Figure 5B). For all tested groups, the qPCR results supported best the relative abundances of bacterial phyla as estimated by primer pair 341f/785r using 454 sequencing technology (Figure 5B). The $\mathrm{qPCR}$ results confirmed that Alphaproteobacteria were overrepresented using primer pair $68 \mathrm{f} / 518 \mathrm{r}$, and underrepresented using 967f/1391r. Bacteroidetes were overrepresented in $967 \mathrm{f} / 1391 \mathrm{r}$ using 454 compared to the qPCR results. Overall, for the taxonomic groups and soil samples tested here, primer pair $341 \mathrm{f} / 785 \mathrm{r}$ is in best accordance with the qPCR results.

\section{DISCUSSION}

To address the need for bacteria-specific primer pairs in order to maximize overall coverage and phylum spectrum for specific applications, we evaluated the performance of four commonly used bacteria-specific primers for short-read sequencing using experimental and in silico approaches on environment-associated microbial communities. All primer sets were found to be highly specific for the domain Bacteria as almost no Archaea and Eukaryotic reads were amplified, and the theoretical coverage for the domains Archaea and Eukaryota was low $(<1.37 \%)$. We demonstrated that primer pair $341 \mathrm{f} / 785 \mathrm{r}$ was able to amplify from a heterogeneous pool of DNA and reveals the highest bacterial taxonomic coverage and diversity. Our study used the latest SILVA database v123 for in silico primer testing and taxonomic identification, and therefore adds value in the field of microbial community analyses as a similar comparative soil bacterial PCR-primer analyses has not been performed in recent 
years. We showed that primer pair 341f/785r performed well on 3 different soil types, including explosives contaminated soil samples, non-contaminated humus-rich forest soil samples and sycamore rhizosphere samples. This suggests a more wide-spread use of primer $341 \mathrm{f} / 785 \mathrm{r}$ in bacterial community studies would enhance the comparability with other studies/environments in which 341f/785r has been commonly used, including mouse cecum samples, lettuce microbiome, bioremediation, and marine habitats. Though, while the study here provides a promising primer set, the long-term value will be in the methodology presented for evaluating primer sets as databases grow and improve, requiring temporal re-evaluation of the performance of primer pairs.

Primer pair 341f/785r had the highest average observed diversity per sample (609 \pm 10 OTUs), significantly higher than the number of OTUs detected by other primer pairs (Figures 1, 2). Our results are therefore in good agreement with the values reported for a clay and loam soil in the UK (879 \pm 21 to $2744 \pm 124$ OTUs), despite the much higher sequencing depth used in their study. The second best primer pair in our study, 967f/1391r, detected $479 \pm 38$ OTUs and this is in line with the results described by Beckers et al. (2016), with an average of 277 OTUs detected per sample for poplar rhizosphere, root, stem and leaf tissues. Primer set 799f/1193r revealed 438 \pm 24 OTUs in the bulk soils compared to $383 \pm 122$ in the A. pseudoplatanus rhizosphere, suggesting that this chloroplast mismatch primer, which was effective in reducing chloroplast amplification for A. pseudoplatanus, comes at a trait-off in the number of OTUs detected and overall coverage and phylum spectrum. Previously, primer set $799 \mathrm{f}$ and $1193 \mathrm{r}$ was shown to effectively reduce chloroplast amplification from $A$. thaliana rhizosphere and root endophytic tissues ((Bulgarelli et al., 2012), (Schlaeppi et al., 2014)), seed endophytes (Truyens et al., 2015), and soybean, canola, and common bean leaves (Copeland et al., 2015). Interestingly, in our study, primer set 967f/1391r was also effective in reducing the co-amplification of chloroplast targets from $A$. pseudoplatanus rhizosphere samples (only 38 reads detected or $<0.1 \%$ ) while maintaining a higher overall coverage compared to 799f/1193r, suggesting it is a promising primer for bacterial community analyses of sycamore trees and potentially other plant species. Other commonly applied strategies to reduce amplification of chloroplast when amplifying microbial communities associated with an eukaryotic host is the use of peptide nucleic acid PCR clamps (Lundberg et al., 2013) or the post-sequence elimination of chloroplast reads given the upgrades of sequencing technology.

A difference noted between primer 341f/785r, 799f/1193r and $967 \mathrm{f} / 1391 \mathrm{r}$ is the two amplicon sizes produced by $341 \mathrm{f} / 785 \mathrm{r}$ (Supplementary Figure 2A) and a potential over-representation of Rhizobiales and Acidobacteria/Subgroup3 (Figure 3A). This effect was not noticed in studies using $341 \mathrm{f} / 785 \mathrm{r}$ on marine microbial communities (Klindworth et al., 2013) or in other studies using the related primer pair 343f (Yergeau et al., 2014, 2015). We confirmed that the dual amplicon sizes were not due to sequencing artifacts, or platform effects (Supplementary Figure 12), but due to gaps in the $16 \mathrm{~S}$ sequence at positions 454-465 and 476-485 (E. coli numbering) in many Alphaproteobacteria not present in the majority of other bacterial taxa (Supplementary Figure 2B). The shorter read length may favor the amplification of these subgroups by primer pair 341f/785r, however we cannot confirm this with the data presently available. Further analyses using shotgun metagenomics could evaluate the extent to which relative abundance differences of these taxa may be a problem. Previously, we used the bacteria/archaea primer pair $515 \mathrm{f}$ 806r to obtain amplicons from the same non-contaminated and TNT-contaminated bulk soil samples to identify the bacteria/archaea microbiome in the mycosphere of the fungus Clitocybe nebularis growing in the TNT-contaminated soil (Thijs, submitted), and we found that 515f/806r produced sequences of 1 length distribution (290 bp average read length), and the relative abundance of the most abundant taxa most closely resembled that generated with 341f/785r (Supplementary Figure 13). Primer pair 515f/806r also amplified archaeal reads as expected $(94.1 \%$ coverage) representing a substantial number of reads in the dataset. An additional advantage of 341f/785r (V3$\mathrm{V} 4$ ) compared to $515 \mathrm{f} / 806 \mathrm{r}$ (V4) is the 1.5 times longer amplicon length and the coverage of two $\mathrm{V}$-regions which can improve species level assignment (Baker et al., 2003; Vasileiadis et al., 2012; Lundberg et al., 2013). Rarefaction analyses also showed a higher phylogenetic diversity and higher number of observed species for 341f/785r compared to 515f/806r (Supplementary Figure 13), suggesting that for bacterial biodiversity studies, primer pair $341 \mathrm{f} / 785 \mathrm{r}$ is more useful for bacterial community analyses by providing increased specificity for bacterial reads.

Principal coordinate analyses of the weighted UniFrac distance matrix revealed that the bulk soil and rhizosphere soil samples clustered per primer pair with high concordance (Figure 3C). Sample type specific differences were also observed from the dual dendrogram-heatmap with the rhizosphere and non-contaminated bulk soil clustering separate from the TNTcontaminated bulk soil samples for all primer pairs (Figure 3D). The relative abundance plots of the most abundant taxa confirmed the observations from the PCoA and heatmap, indicating significant differences in relative abundance across the phylum to genus level, suggesting primer-pair dependent amplification biases (Figure 3A). Notably, primer pair $68 \mathrm{f} / 515 \mathrm{r}$ showed a large overrepresentation of Alphaproteobacteria and less coverage of all other taxa compared to the other primer pairs. The likely explanation for this observation is the poor predicted in silico coverage of primer pair $68 \mathrm{f} / 518 \mathrm{r}$ for many taxa, leading to the preferential amplification of certain groups, while the other primer sets covered a larger number of phyla resulting in an increased number of reads that successfully clustered against the SILVA database.

Based on the in silico coverage and PrimerProspector score, primer pair 341f/785r scored best with $96.51 \%$ coverage of the domain Bacteria, and $0.07 \pm 0.0005$ and $0.1 \pm 0.0006$ average weighted scores for $341 \mathrm{f}$ and $785 \mathrm{r}$, respectively (Table 2). For the in silico domain coverage prediction we used the weighted scoring in PrimerProspector and the latest SSU non-redundant reference SILVA database v123. Primer pair 341f/785r was developed against an earlier version of the SILVA database (release 108); here we show that the primer pair still attains a 
high taxonomic sensitivity against the updated SILVA database containing an additional 222,034 new sequences since 2013. The PrimerProspector score of $68 \mathrm{f}(0.71 \pm 0.002)$ and $518 \mathrm{r}(0.08$ \pm 0.0005 ) was less optimal than $341 \mathrm{f} / 785 \mathrm{r}$, and this could be in part explained by the fact that $3 \%$ of the sequences had a mismatch in the last $3^{\prime}$ base of primer $68 \mathrm{f}$ which detrimentally affects amplification. For example, Chlorobi, an ubiquitous taxon in soil, had in $40 \%$ of the sequences a mismatch in the $3^{\prime}$ region of the $16 \mathrm{~S}$ rRNA gene targeted by $68 \mathrm{f}$, and this resulted in a complete absence of amplification. Next to Chlorobi, $68 \mathrm{f} / 518 \mathrm{r}$ did not cover many other important groups in soil (Supplementary Table 4B) and this was reflected in the lowest Shannon diversity (ranging from 3.2 to 5.4) and number of OTUs (214 \pm 5 ) for all soil samples. Previously, primer pair $68 \mathrm{f} / 518 \mathrm{r}$ was used to amplify iron-oxidizing Zetaproteobacteria in soil but it has not been extensively validated as a primer for soil microbial community characterization (McAllister et al., 2011). Because primer $68 \mathrm{f} / 515 \mathrm{r}$ cannot reliably assess Alphaproteobacteria and has a low coverage, we do not recommend this primer for soil microbiome analyses. For comparison, primer pair 799f/1193r $(0.37 \pm 0.001,0.21 \pm 0.0006)$ had a better PrimerProspector score, while $967 \mathrm{f} / 1391$ had the worst score $(0.32 \pm 0.0007$ and $0.87 \pm 0.002$ ), though experimental results did not suggest that $967 \mathrm{f} / 1391 \mathrm{r}$ performed the worst in terms of phylogenetic richness. For instance, in silico prediction suggested a high non-coverage for Bacteroidetes (Figure 4), but experimentally 967f/1391r detected significantly the highest relative abundance of Bacteroidetes, suggesting that care should be taken to interpret in silico analyses and experimental analyses are essential to support the findings.

The results obtained by 454 technology were also compared with qPCR analyses of the same soil samples to test whether bulk amplicon sequencing appropriately quantified the relative abundance of specific OTUs in the community (Figure 5). Primer pair 341f $/ 785 \mathrm{r}$ approximated best the qPCR data using group-specific qPCR primer sets (Pfeiffer et al., 2014), followed by $967 \mathrm{f} / 1391 \mathrm{r}$ and $799 \mathrm{f} / 1193 \mathrm{r}$. Indeed, the ratio between the bacterial 16S rRNA gene copies as determined by qPCR supported the relative amount of bacterial phyla as estimated from the 454 amplicon library (Figure 5), indicating that $341 \mathrm{f} / 785 \mathrm{r}$ used for the 454-sequencing allowed a high rate of coverage and amplified with relatively little bias under the applied conditions. Additionally, further analyses, such as with mock communities, could evaluate the extent to which the primer pairs truly represent microbial community distributions. In addition, previous shotgun metagenome analyses have shown that $341 \mathrm{f} / 785 \mathrm{r}$ reflects metagenome libraries (Klindworth et al., 2013).

In addition to presenting an approach to evaluate the performance of primer pairs to characterize soil bacterial microbiomes, our study also provides the first view of bacterial communities present at a TNT-contaminated forest soil (Figure 3A). The forest soil was dominated by Alphaproteobacteria (37.7\%), Bacteroidetes (12.5\%), Gammaproteobacteria (9.9\%), Betaproteobacteria (9.6\%), Acidobacteria (8.5\%), and Actinobacteria (4.1\%), an observation made using data generated from all four primer pairs. The
TABLE 2 | Percentage of sequences with last $3^{\prime}$ base mismatch and PrimerProspector score.

\begin{tabular}{lcc}
\hline Primer pair & Last base mismatch $(\%)$ & Average score \pm SE \\
\hline $341 f$ & 0.92 & $0.07 \pm 0.0005$ \\
$518 r$ & 0.41 & $0.08 \pm 0.0005$ \\
$785 r$ & 0.79 & $0.1 \pm 0.0006$ \\
$1193 r$ & 0.48 & $0.21 \pm 0.0006$ \\
$967 f$ & 0.34 & $0.32 \pm 0.0007$ \\
$799 f$ & 5.29 & $0.37 \pm 0.001$ \\
$68 f$ & 3.19 & $0.71 \pm 0.002$ \\
$1391 r$ & 4.08 & $0.87 \pm 0.002$ \\
\hline
\end{tabular}

The SILVA SSU non-redundant database release 123 was used for the analyses, which contains 597,607 bacterial sequences.

TNT-polluted soil harbored a bacterial community that shifted toward a higher relative abundance of the Gammaproteobacteria (LDA $>4.5, p<0.05)$ compared to the non-contaminated bulk soil (Supplementary Figure 8). Although different primer pairs indicated different dominant OTUs, a few bacterial groups were commonly identified as more abundant in the TNTcontaminated bulk soil including Acidobacteria/Subgroup1, Acidobacteriales, Mizugakiibacter, and Rhodanobacter. Primer pair 341f/785R detected an enrichment in the TNTpolluted soil of Xanthomonadaceae (Gammaproteobacteria), Bradyrhizobiaceae, Caulobacteracae, and alphaIcluster of the Rhizobiales (Alphaproteobacteria), and Chitinophagaceae (Bacteroidetes), suggesting these taxa are quite tolerant to high TNT-concentrations ( $>5 \%)$. A higher prevalence of Gamma- and Alpha-proteobacteria has been reported in a hydrocarbon contaminated soil (Yergeau et al., 2014), which suggest an r-strategy life-style for these taxa. With respect to TNT-contaminated soil, some of the groups detected in this study are in agreement with the results from clone libraries and DGGE-analyses of TNT-contaminated soil in the UK (Travis et al., 2008), and France (Eyers et al., 2006). Moreover, cultured strains of the Gamma- and Alpha-proteobacteria (Rhizobiales) have the ability to reduce trinitrotoluene to aminonitrotoluene (Labidi et al., 2001; Ramos et al., 2005; Chien et al., 2014). Importantly, we detected recently that the soil bacterium Raoultella (Enterobacteriaceae) has the ability to liberate nitrite from TNT for growth catalyzed by a protein of the Old Yellow Enzyme family, N-ethylmaleimide reductase (Thijs et al., 2014). The other taxa in this study which appear to have been enriched on TNT-contaminated soil have not been investigated in cultureconditions, and constitute promising candidates for further investigation.

\section{CONCLUSION}

Using experimental and in silico approaches for bacterial community characterization, we found that among the four bacteria-specific primer pairs tested in this study, 341f/785r is the most promising set for bacterial community analyses because it showed the highest taxonomic coverage, diversity, 
reproducibility, PCR-amplification efficiency and best agreement with qPCR data on the tested soil samples. The primers used in this study are also applicable to be used on the Illumina platform and Ion Torrent. Knowledge on how various primer pairs compare and amplify taxa is of critical importance and must be considered as part of a robust experimental design. Together temporal re-evaluation of primer pairs against upto-date databases is important in order to best represent our understanding of microbial taxonomy.

\section{AUTHOR CONTRIBUTIONS}

Based on his experience in primer design and testing, MO contributed significantly in the design of the work and critical revision of the article. BB, STr, and VS were of great help for the practical design of the work including next generation sequencing library preparation, and data collection. STh designed the work, performed data collection and interpretation, and wrote the paper. JDV has great experience in sequencing data analyses and helped with the interpretation and critical revision

\section{REFERENCES}

Apprill, A., McNally, S., Parsons, R., and Weber, L. (2015). Minor revision to V4 region SSU rRNA 806R gene primer greatly increases detection of SAR11 bacterioplankton. Aquat. Microb. Ecol. 75, 129-137. doi: 10.3354/ame01753

Baker, G. C., Smith, J. J., and Cowan, D. A. (2003). Review and reanalysis of domain-specific $16 \mathrm{~S}$ primers. J. Microbiol. Methods 55, 541-555. doi: 10.1016/j.mimet.2003.08.009

Beckers, B., Op De Beeck, M., Weyens, N., Van Acker, R., Van Montagu, M., Boerjan, W., et al. (2016). Lignin engineering in field-grown poplar trees affects the endosphere bacterial microbiome. Proc. Natl. Acad. Sci. U.S.A. 113, 2312-2317. doi: 10.1073/pnas. 1523264113

Bell, T. H., Cloutier-Hurteau, B., Al-Otaibi, F., Turmel, M. C., Yergeau, E., Courchesne, F., et al. (2015). Early rhizosphere microbiome composition is related to the growth and $\mathrm{Zn}$ uptake of willows introduced to a former landfill. Environ. Microbiol. 17, 3025-3038. doi: 10.1111/1462-2920.12900

Bell, T. H., Yergeau, E., F Juck, D., G. Whyte, L and W. Greer, C. (2013a). Alteration of microbial community structure affects diesel biodegradation in an Arctic soil. FEMS Microbiol. Ecol. 85, 51-61. doi: 10.1111/1574-6941. 12102

Bell, T. H., Yergeau, E., Maynard, C., Juck, D., Whyte, L, G., and Greer, C., W. (2013b). Predictable bacterial composition and hydrocarbon degradation in Arctic soils following diesel and nutrient disturbance. ISME J. 7, 1200-1210. doi: 10.1038/ismej.2013.1

Berg, G., Krause, R., and Mendes, R. (2015). Cross-kingdom similarities in microbiome ecology and biocontrol of pathogens. Front. Microbiol. 6:1311. doi: $10.3389 /$ fmicb. 2015.01311

Berry, D., Ben Mahfoudh, K., Wagner, M., and Loy, A. (2011). Barcoded primers used in multiplex amplicon pyrosequencing bias amplification. Appl. Environ. Microbiol. 77, 7846-7849. doi: 10.1128/AEM.05220-11

Bodenhausen, N., Horton, M. W., and Bergelson, J. (2013). Bacterial communities associated with the leaves and the roots of Arabidopsis thaliana. PLoS ONE 8:e56329. doi: 10.1371/journal.pone.0056329

Bulgarelli, D., Rott, M., Schlaeppi, K., Ver Loren van Themaat, E., Ahmadinejad, N., Assenza, F., et al. (2012). Revealing structure and assembly cues for Arabidopsis root-inhabiting bacterial microbiota. Nature 488, 91-95. doi: 10.1038/nature11336

Caporaso, J. G., Bittinger, K., Bushman, F. D., DeSantis, T. Z., Andersen, G., L., and Knight, R. (2010a). PyNAST: a flexible tool for aligning sequences to a template alignment. Bioinformatics 26, 266-267. doi: 10.1093/bioinformatics/btp636 of the article, and English language correction. JV and NW are authorities in the field of bioremediation research and both gave their approval and critical evaluation of the final version to be published.

\section{ACKNOWLEDGMENTS}

This work was financed by the Methusalem project 08M03VGRJ and was also supported by grants to STh and NW from the Fund of Scientific Research Flanders. Thanks to Prof. Dr. GeertJan Bex for help with setting up the Flemish Supercomputer Centre (VSC) supported by the Research FoundationFlanders (FWO) in partnership with the five Flemish university associations.

\section{SUPPLEMENTARY MATERIAL}

The Supplementary Material for this article can be found online at: http://journal.frontiersin.org/article/10.3389/fmicb. 2017.00494/full\#supplementary-material

Caporaso, J. G., Kuczynski, J., Stombaugh, J., Bittinger, K., Bushman, F. D., Costello, E. K., et al. (2010b). QIIME allows analysis of highthroughput community sequencing data. Nat. Methods 7, 335-336. doi: 10.1038/nmeth.f.303

Caporaso, J. G., Lauber, C. L., Walters, W. A., Berg-Lyons, D., Lozupone, C. A., Turnbaugh, P. J., et al. (2011). Global patterns of $16 \mathrm{~S}$ rRNA diversity at a depth of millions of sequences per sample. Proc. Natl. Acad. Sci. U.S.A 108(Suppl. 1), 4516-4522. doi: 10.1073/pnas.1000080107

Chapman, H. D. (1965). “Cation-exchange capacity," in Methods of Soil Analysis Chemical and Microbiological Properties, Vol. 9, ed C. A. Black (New York, NY: Agronomy), 891-901.

Chelius, M. K., and Triplett, E. W. (2001). The diversity of archaea and bacteria in association with the roots of Zea mays L. Microb. Ecol. 41, 252-263. doi: $10.1007 / \mathrm{s} 002480000087$

Chien, C. C., Kao, C. M., Chen, D. Y., Chen, S. C., and Chen, C. C. (2014). Biotransformation of trinitrotoluene (TNT) by Pseudomonas spp. isolated from a TNT-contaminated environment. Environ. Toxicol. Chem. 33, 1059-1063. doi: 10.1002/etc. 2553

Colwell, R. K., Chao, A., Gotelli, N. J., Lin, S. Y., Mao, C. X., Chazdon, R. L., et al. (2012). Models and estimators linking individual-based and sample-based rarefaction, extrapolation and comparison of assemblages. J. Plant Ecol. 5, 3-21. doi: $10.1093 /$ jpe/rtr044

Copeland, J. K., Yuan, L., Layeghifard, M., Wang, P. W., and Guttman, D. S. (2015). Seasonal community succession of the phyllosphere microbiome. Mol. Plant Microbe Interact. 28, 274-285. doi: 10.1094/MPMI-10-14-0331-FI

Culman, S. W., Snapp, S. S., Freeman, M. A., Schipanski, M. E., Beniston, J., Lal, R., et al. (2012). Permanganate oxidizable carbon reflects a processed soil fraction that is sensitive to management. Soil Sci. Soc. Am. J. 76, 494-504. doi: 10.2136/sssaj2011.0286

Edgar, R. C., Haas, B. J., Clemente, J. C., Quince, C., and Knight, R. (2011). UCHIME improves sensitivity and speed of chimera detection. Bioinformatics 27, 2194-2200. doi: 10.1093/bioinformatics/btr381

Eyers, L., Smoot, J. C., Smoot, L. M., Bugli, C., Urakawa, H., McMurry, Z., et al. (2006). Discrimination of shifts in a soil microbial community associated with TNT-contamination using a functional ANOVA of 16S rRNA hybridized to oligonucleotide microarrays. Environ. Sci. Technol. 40, 5867-5873. doi: 10.1021/es0609093

Felsenstein, J. (2003). Inferring Phylogenies. Sunderland, MA: Sinauer Associates.

Ghyselinck, J., Pfeiffer, S., Heylen, K., Sessitsch, A., and De Vos, P. (2013). The effect of primer choice and short read sequences on the 
outcome of $16 \mathrm{~S}$ rRNA gene based diversity studies. PLoS ONE8: e71360. doi: 10.1371/journal.pone.0071360

Gilbert, J. A., Jansson, J. K., and Knight, R. (2014). The Earth Microbiome project: successes and aspirations. BMC Biol. 12:69. doi: 10.1186/s12915-014-0069-1

Hart, M. M., Aleklett, K., Chagnon, P. L., Egan, C., Ghignone, S., Helgason, T., et al. (2015). Navigating the labyrinth: a guide to sequence-based, community ecology of arbuscular mycorrhizal fungi. New Phytol. 207, 235-247. doi: $10.1111 / \mathrm{nph} .13340$

Hiergeist, A., Reischl, U., and Gessner, A. (2016). Multicenter quality assessment of $16 \mathrm{~S}$ ribosomal DNA-sequencing for microbiome analyses reveals high inter-center variability. Int. J. Med. Microbiol. 306, 334-342. doi: 10.1016/j.ijmm.2016.03.005

Hug, L. A., Baker, B. J., Anantharaman, K., Brown, C. T., Probst, A. J., Castelle, C. J., et al. (2016). A new view of the tree of life. Nat. Microbiol. 1:16048. doi: 10.1038/nmicrobiol.2016.48

Jenkins, J. R., Viger, M., Arnold, E. C., Harris, Z. M., Ventura, M., Miglietta, F., et al. (2016). Biochar alters the soil microbiome and soil function: results of nextgeneration amplicon sequencing across Europe. Global Change Biol. Bioenergy 9, 591-612. doi: 10.1111/gcbb.12371

Klindworth, A., Pruesse, E., Schweer, T., Peplies, J., Quast, C., Horn, M., et al. (2013). Evaluation of general 16S ribosomal RNA gene PCR primers for classical and next-generation sequencing-based diversity studies. Nucleic Acids Res. 41:e1. doi: 10.1093/nar/gks808

Labidi, M., Ahmad, D., Halasz, A., and Hawari, J. (2001). Biotransformation and partial mineralization of the explosive 2,4,6-trinitrotoluene (TNT) by rhizobia. Can. J. Microbiol. 47, 559-566. doi: 10.1139/w01-040

Lee, T. K., Van Doan, T., Yoo, K., Choi, S., Kim, C., and Park, J. (2010). Discovery of commonly existing anode biofilm microbes in two different wastewater treatment MFCs using FLX Titanium pyrosequencing. Appl. Microbiol. Biotechnol. 87, 2335-2343. doi: 10.1007/s00253-010-2680-6

Lozupone, C. A., and Knight, R. (2007). Global patterns in bacterial diversity. Proc. Natl. Acad. Sci. U.S.A. 104, 11436-11440. doi: 10.1073/pnas.0611525104

Lundberg, D. S., Lebeis, S. L., Paredes, S. H., Yourstone, S., Gehring, J., Malfatti, S., et al. (2012). Defining the core Arabidopsis thaliana root microbiome. Nature 488, 86-90. doi: 10.1038/nature 11237

Lundberg, D. S., Yourstone, S., Mieczkowski, P., Jones, C. D., and Dangl, J. L. (2013). Practical innovations for high-throughput amplicon sequencing. Nat. Methods 10, 999-1002. doi: 10.1038/nmeth.2634

Mahnert, A., Moissl-Eichinger, C., and Berg, G. (2015). Microbiome interplay: plants alter microbial abundance and diversity within the built environment. Front. Microbiol. 6:887. doi: 10.3389/fmicb.2015.00887

McAllister, S. M., Davis, R. E., McBeth, J. M., Tebo, B. M., Emerson, D., and Moyer, C. L. (2011). Biodiversity and emerging biogeography of the neutrophilic iron-oxidizing Zetaproteobacteria. Appl. Environ. Microbiol. 77, 5445-5457. doi: 10.1128/AEM.00533-11

Oliveros, J. C. (2007). VENNY. An Interactive Tool for Comparing Lists with Venn Diagrams. Available online at: http://bioinfogp.cnb.csic.es/tools/venny

Parada, A. E., Needham, D., M., and Fuhrman, J., A. (2015). Every base matters: assessing small subunit rRNA primers for marine microbiomes with mock communities, time series and global field samples. Environ. Microbiol. 18, 1403-1414. doi: 10.1111/1462-2920.13023

Parks, D. H., Tyson, G. W., Hugenholtz, P., and Beiko, R. G. (2014). STAMP: statistical analysis of taxonomic and functional profiles. Bioinformatics 30, 3123-3124. doi: 10.1093/bioinformatics/btu494

Paulson, J. N., Stine, O. C., Bravo, H. C., and Pop, M. (2013). Differential abundance analysis for microbial marker-gene surveys. Nat. Methods 10, 1200-1202. doi: 10.1038/nmeth.2658

Pfeiffer, S., Pastar, M., Mitter, B., Lippert, K., Hackl, E., Lojan, P., et al. (2014). Improved group-specific primers based on the full SILVA 16S rRNA gene reference database. Environ. Microbiol. 16, 2389-2407. doi: $10.1111 / 1462-2920.12350$

Price, M. N., Dehal, P. S., and Arkin, A. P. (2009). FastTree: computing large minimum evolution trees with profiles instead of a distance matrix. Mol. Biol. Evol. 26, 1641-1650. doi: 10.1093/molbev/msp077

Quast, C., Pruesse, E., Yilmaz, P., Gerken, J., Schweer, T., Yarza, P., et al. (2013). The SILVA ribosomal RNA gene database project: improved data processing and web-based tools. Nucleic Acids Res. 41, D590-D596. doi: 10.1093/nar/ gks1219
Ramos, J. L., Gonzalez-Perez, M. M., Caballero, A., and van Dillewijn, P. (2005). Bioremediation of polynitrated aromatic compounds: plants and microbes put up a fight. Curr. Opin. Biotechnol. 16, 275-281. doi: 10.1016/j.copbio.2005.03.010

Robertson, C. E., Harris, J. K., Wagner, B. D., Granger, D., Browne, K., Tatem, B., et al. (2013). Explicet: graphical user interface software for metadata-driven management, analysis and visualization of microbiome data. Bioinformatics 29, 3100-3101. doi: 10.1093/bioinformatics/btt526

Schlaeppi, K., Dombrowski, N., Oter, R., Ver Loren van Themaat, E., and SchulzeLefert, P. (2014). Quantitative divergence of the bacterial root microbiota in Arabidopsis thaliana relatives. Proc. Natl. Acad. Sci. U.S.A. 111, 585-592. doi: $10.1073 /$ pnas. 1321597111

Segata, N., Izard, J., Waldron, L., Gevers, D., Miropolsky, L., Garrett, W. S., et al. (2011). Metagenomic biomarker discovery and explanation. Genome Biol. 12:R60. doi: 10.1186/gb-2011-12-6-r60

Soergel, D. A., Dey, N., Knight, R., and Brenner, S. E. (2012). Selection of primers for optimal taxonomic classification of environmental $16 \mathrm{~S}$ rRNA gene sequences. ISME J. 6, 1440-1444. doi: 10.1038/ismej.20 11.208

Sogin, M. L., Morrison, H. G., Huber, J. A., Welch, D. M., Huse, S. M., Neal, P. R., et al. (2006). Microbial diversity in the deep sea and the underexplored "rare biosphere". Proc. Nat. Acad. Sci. 103, 12115-12120. doi: 10.1073/pnas.0605127103

Thijs, S., Sillen, W., Rineau, F., Weyens, N., and Vangronsveld, J. (2016). Towards an enhanced understanding of plant-microbiome interactions to improve phytoremediation: engineering the metaorganism. Front. Microbiol. 7:341. doi: 10.3389/fmicb.2016.00341

Thijs, S., Van Hamme, J., Gkorezis, P., Rineau, F., Weyens, N., and Vangronsveld, J. (2014). Draft genome sequence of Raoultella ornithinolytica tnt, a trinitrotoluene-denitrating and plant growth-promoting strain isolated from explosive-contaminated soil. Genome Announc. 2:e00491-14. doi: 10.1128/genomeA.00491-14

Travis, E. R., Bruce, N. C., and Rosser, S. J. (2008). Short term exposure to elevated trinitrotoluene concentrations induced structural and functional changes in the soil bacterial community. Environ. Pollut. 153, 432-439. doi: 10.1016/j.envpol.2007.08.006

Truyens, S., Beckers, B., Thijs, S., Weyens, N., Cuypers, A., and Vangronsveld, J. (2015). The effects of the growth substrate on cultivable and total endophytic assemblages of Arabidopsis thaliana. Plant Soil 405, 325-336. doi: 10.1007/s11104-015-2761-5

Truyens, S., Weyens, N., Cuypers, A., and Vangronsveld, J. (2013). Changes in the population of seed bacteria of transgenerationally Cd-exposed Arabidopsis thaliana. Plant Biol. 15, 971-981. doi: 10.1111/j.1438-8677.2012.00711.x

Vasileiadis, S., Puglisi, E., Arena, M., Cappa, F., Cocconcelli, P., S., and Trevisan, M. (2012). Soil bacterial diversity screening using single 16S rRNA gene V regions coupled with multi-million read generating sequencing technologies. PLoS ONE 7:e42671. doi: 10.1371/journal.pone.0042671

Walker, J. J., and Pace, N. R. (2007). Phylogenetic composition of rocky mountain endolithic microbial ecosystems. Appl. Environ. Microbiol. 73, 3497-3504. doi: 10.1128/AEM.02656-06

Walters, W. A., Caporaso, J. G., Lauber, C. L., Berg-Lyons, D., Fierer, N., and Knight, R. (2011). PrimerProspector: de novo design and taxonomic analysis of barcoded polymerase chain reaction primers. Bioinformatics 27, 1159-1161. doi: 10.1093/bioinformatics/btr087

Walters, W., Hyde, E. R., Berg-Lyons, D., Ackermann, G., Humphrey, G., Parada, A., et al. (2016). Improved bacterial 16S rRNA gene (V4 and V4-5) and fungal internal transcribed spacer marker gene primers for microbial community surveys. mSystems 1, e00009-00015. doi: 10.1128/mSystems.00009-15

Wu, J. Y., Jiang, X. T., Jiang, Y. X., Lu, S. Y., Zou, F., and Zhou, H. W. (2010). Effects of polymerase, template dilution and cycle number on PCR based $16 \mathrm{~S}$ rRNA diversity analysis using the deep sequencing method. BMC Microbiol. 10:255. doi: 10.1186/1471-2180-10-255

Yang, S., Wen, X., Jin, H., and Wu, Q. (2012). Pyrosequencing investigation into the bacterial community in permafrost soils along the China-Russia Crude Oil Pipeline (CRCOP). PLOS ONE 7:e52730. doi: 10.1371/journal.pone.00 52730

Yergeau, E., Bell, T. H., Champagne, J., Maynard, C., Tardif, S., Tremblay, J., et al. (2015). Transplanting soil microbiomes leads to lasting effects on willow 
growth, but not on the rhizosphere microbiome. Front. Microbiol. 6:1436. doi: 10.3389/fmicb.2015.01436

Yergeau, E., Lawrence, J. R., Sanschagrin, S., Waiser, M. J., Korber, D. R., and Greer, C. W. (2012b). Next-generation sequencing of microbial communities in the Athabasca River and its tributaries in relation to oil sands mining activities. Appl. Environ. Microbiol. 78, 7626-7637. doi: 10.1128/AEM.02036-12

Yergeau, E., Sanschagrin, S., Beaumier, D., and Greer, C. W. (2012a). Metagenomic analysis of the bioremediation of diesel-contaminated Canadian high arctic soils. PLoS ONE 7:e30058. doi: 10.1371/journal.pone.0030058

Yergeau, E., Sanschagrin, S., Maynard, C., St-Arnaud, M., and Greer, C. W. (2014). Microbial expression profiles in the rhizosphere of willows depend on soil contamination. ISME J. 8, 344-358. doi: 10.1038/ismej.2013.163
Conflict of Interest Statement: The authors declare that the research was conducted in the absence of any commercial or financial relationships that could be construed as a potential conflict of interest.

Copyright ( 2017 Thijs, Op De Beeck, Beckers, Truyens, Stevens, Van Hamme, Weyens and Vangronsveld. This is an open-access article distributed under the terms of the Creative Commons Attribution License (CC BY). The use, distribution or reproduction in other forums is permitted, provided the original author(s) or licensor are credited and that the original publication in this journal is cited, in accordance with accepted academic practice. No use, distribution or reproduction is permitted which does not comply with these terms. 